\title{
Olfactory Ensheathing Cells and the Structure of the Primary Olfactory Projection
}

\author{
A thesis \\ submitted by \\ Amir Ali Badiei \\ In partial fulfillment of the requirements \\ for the degree of \\ Master of Science in Biomedical Sciences
}

at

TUFTS UNIVERSITY

SCHOOL OF MEDICINE

Public Health \& Professional Degree Programs

Date

August, 2010

ADVISER: James E. Schwob, MD, PhD

READER: Karina Meiri, $\mathrm{PhD}$ 


\begin{abstract}
Olfactory ensheathing cells (OECs) are the glial cells that accompany axons from the olfactory epithelium to their targets in the olfactory bulb. They possess unique features that have made them the subject of much study as being potentially useful in cell-based therapeutic approaches to CNS repair.

Investigation of OECs has demonstrated antigenic and morphological heterogeneity in their population. No marker specific to and selective for OECs have yet been identified, and many of the markers used are variably expressed by other glial cells. Even among OECs, these markers appear to vary in vivo (depending on their anatomical location, contact with other cells, and developmental timing) and in vitro. The variation across the population of OECs has compromised their isolation and characterization. It has also made the task of identifying meaningful subpopulations - with greater or lesser therapeutic utility - dependant on identifying the source of their variability. Such information would aid in both the harvest and experimental manipulation of OECs to optimize their therapeutic effect.
\end{abstract}

One way to understand the nature of this variability is to seek its potential causes in vivo. This must begin with an examination of the structure of the olfactory nerve. Here, the structure and development of the primary olfactory projection are thoroughly reviewed with an emphasis on OECs and the cells with which they make contact. The relevant experimental results are also discussed. The weight of anatomical evidence indicates that the structural variations described in different locations and across species are mostly the result of spatiotemporal developmental factors. As such, the formation of the olfactory projection is mediated primarily by the source tissue, the olfactory epithelium. Cell-autonomous development is common elsewhere, and suits the evolutionary age and importance of olfaction, as well as its continued regenerative capacity. Our findings provide a more systematic anatomical understanding of this nerve. That understanding indicates that variation in axon ensheathment by OECs is an inherent feature arising from the flexibility of the ensheathing program. This perspective, along with the anatomical data reviewed here, can inform more carefully controlled laboratory investigations designed to uncover the detailed mechanisms governing OEC biology. 
I would like to thank Dr. James Schwob for his valued mentorship, Dr. Karina Meiri for her enthusiasm and support as reader and friend, and all of the members of the Schwob and Meiri Labs for welcoming me as I completed this thesis. In particular, Nikolai Schnittke, Richard Krolewski, Dr. Eric Holbrook, and Adam Packard, for their provision of extensive bits of wisdom regarding olfactory anatomy and molecular biology. Also, Dr. Kathleen Yee, Dr. Woochan Jang, Dr. Daniel Flis, Dave Wu, Po Tse, and Barbara Cohen, for being the kind and supportive people I am fortunate to have worked with every day.

Of the many researchers whose work I have here reviewed, I would like to pay special respect to Dr. Ron Doucette of the University of Saskatchewan, Saskatoon, Canada. His extraordinary diligence is to be admired. 


\section{Table of Contents}

1. Overview 1

2. Epithelium 4

2.1. What pioneers the pathway: OECs, olfactory axons, or both? 8

2.2. Beyond the Epithelium 15

3. Lamina Propria 16

3.1. LEOB Merger 19

3.2. How would cooperative ensheathment arise? 21

3.3. Olfactory Nerve Fibroblasts 24

3.4. Fascicle Merger 28

3.5. Are there interspecies differences in ensheathment style? 31

3.5.1. Interspecies Cooperative Ensheathment 32

3.5.2. Interspecies Nuclear Location 34

3.6. How is OEC nuclear location determined? 37

3.7. Primacy of Merger 41

4. Subarachnoid Region 42

5. Olfactory Nerve Layer 46

5.1. Laminarity: Axon Sorting 49

5.2. Why is there such a distinct ONLo? 55

6. Glomerular Layer 61

7. Endnotes 63

8. References 64 


\section{List of Figures}

Figure 1 (Mombaerts, 1996, Fig. 2F) 2

Figure 2 (Modified from Farbman, 1992, Fig. 2-18) 3

Figure 3 (Modified from Franssen, 2007, Fig. 1) 4

Figure 4 (Modified from Farbman, 1992, Fig. 2-11) 4

Figure 5 (Johnson, 2000, Fig. 2A) 5

Figure 6 (Li, 2005, Fig. 3A) 5

Figure 7 (Holbrook, 1995, Fig. 10B) 6

Figure 8 (Li, 2005, Fig. 3C) 6

Figure 9 (Modified from Holbrook, 1995, Fig. 8) 6

Figure 10 (Tennent \& Chuah, 1996, Fig. 2A) 10

Figure 11 (Liu, 1995, Figs. 5AB, 6AB) 13

Figure 12 (Modified from Field, 2003, Fig. 1C) 17

Figure 13 (Modified from Li, 2005b, Fig. 4) 17

Figure 14 (Rafols \& Getchell, 1983, Fig. 16C) 17

Figure 15 (Li, 2005, Fig. 4) 18

Figure 16 (Holbrook, 2005, Fig. 1A) 18

Figure 17 (Modified from Field, 2003, Fig. 2) 20

Figure 18 (Schwob, 1992, Fig. 10C) 26

Figure 19 (Holbrook, 2005, Fig. 6A) 26

Figure 20 (Herrera, 2005, Fig. 4A) 26

Figure 21 (Holbrook, 2005, Fig. 6B) 27

Figure 22 (Schwob, 1992, Fig. 10C) 27

Figure 23 Diagram of forms of merger in the lamina propria 29

Figure 24 (Kawaja, 2009, Fig. 2) 31

Figure 25 (Smithson, Kawaja, 2009, Fig. 1C) 33

Figure 26 (Kawaja, 2009, Figs. 4AB) 33

Figure 27 (Boyd, 2003, Fig. 2A) 35

Figure 28 (Barber \& Lindsay, 1982, Fig. 10) 35

Figure 29 (Doucette, 1983a, Fig. 9) 35

Figure 30 (Herrera, 2005, Fig. 4B) 36

Figure 31 (Raisman, 1985, Fig. 4) 36

Figure 32 (Modified from Graziadei, 1973, Fig. 1) 36 
Figure 33 (Cuschieri \& Bannister, 1975b, Fig. 23) 38

Figure 34 (Farbman \& Squinto, 1985, Fig. 2) 38

Figure 35 (Marin-Padilla \& Amieva, 1989, Fig. 2C) 38

Figure 36 (Doucette, 1991, Fig. 11) 42

Figure 37 (Doucette, 1993a, Fig. 12) 45

Figure 38 (Modified from Skinner, 2005, Fig. 5B) 45

Figure 39 (Doucette, 1991, Fig. 8A) 45

Figure 40 (Valverde, 1991, Fig. 6) 47

Figure 41 (Au, 2002, Fig. 7) 50

Figure 42 (Au, 2002, Fig. 8) 50

Figure 43 (Treloar, 2002, Fig. 1A) 51

Figure 44 (Treloar, 2002, Figs. 2AB) 51

Figure 45 (Potter, 2001, Animation 3, Frame 7) 51

Figure 46 (Potter, 2001, Animation 3, Frame 15) 51

Figure 47 (Mombaerts, 1996, Fig. 2D) 52

Figure 48 (Graziadei \& Monti-Graziadei, 1986, Fig. 7) 54

Figure 49 (Windus, 2010, Fig. 1B) 56

Figure 50 (Windus, 2010, Fig. 1C) 56

Figure 51 (Marin-Padilla \& Amieva, 1989, Fig. 6) 60

Figure 52 (Marin-Padilla \& Amieva, 1989, Fig. 7) 60 


\section{List of Abbreviations}

OSN olfactory sensory neuron

SUS Sustentacular cell

MVC microvillar cell

GBC globose basal cell

HBC horizontal basal cell

OEC olfactory ensheathing cell

pOEC progenitor olfactory ensheathing cell

$\mathrm{p} / \mathrm{OEC} \quad$ transitional progenitor-mature olfactory ensheathing cell

EOB ensheathed olfactory bundle

LEOB laminated ensheathed olfactory bundle

ONF olfactory nerve fibroblast

LMC leptomeningeal cell

ONL (o/i) olfactory nerve layer (outer/inner)

GL glomerular layer

\section{Definitions}

fascicle $\quad$ LEOB(s) embedded in endoneurium and surrounded by perineurial ONF processes

rootlet LEOB surrounded by LMC processes 


\section{Olfactory Ensheathing Cells and the Structure of the Primary Olfactory Projection}

\section{1 - Overview}

The cell bodies of olfactory sensory neurons (OSN) reside within the olfactory epithelium, which forms the lining of the posteriodorsal nasal cavity. Their small-diameter axons traverse the epithelial basal lamina and travel dorsally and caudally through the lamina propria towards the cribriform plate of the ethmoid bone. Immediately dorsal to the cribriform plate sits the CNS target of the olfactory axons, the olfactory bulb (OB, bulb). The cribriform plate is perforated by multiple foramina through which the axons enter the cranial cavity, pass through the meninges, and contact the bulb.

The most superficial layer of the bulb is the olfactory nerve layer (ONL), where the arriving axons rearrange and take aim towards their specific targets in the underlying glomerular layer (GL). The glomerular layer consists of thousands of spherical neuronal pockets called glomeruli where the primary olfactory axons synapse on the dendrites of mitral and tufted cells (the relay neurons of the bulb), and juxtaglomerular cells (interneurons that form the walls of the glomeruli). Each glomerulus is innervated by axons expressing the same odorant receptor (OR), and each bulb has two glomeruli - one medial and one lateral - innervated by axons of OSNs that express the same OR (Mombaerts, 1996).

This review focuses on the glial cells - olfactory ensheathing cells (OECs) - that accompany the primary olfactory axons from their origin in the epithelium to their synapses in the glomerular layer. As such, the structural details of the deeper laminae of the bulb - the external plexiform, mitral cell, internal plexiform, and granule cell layers - will not be discussed. 


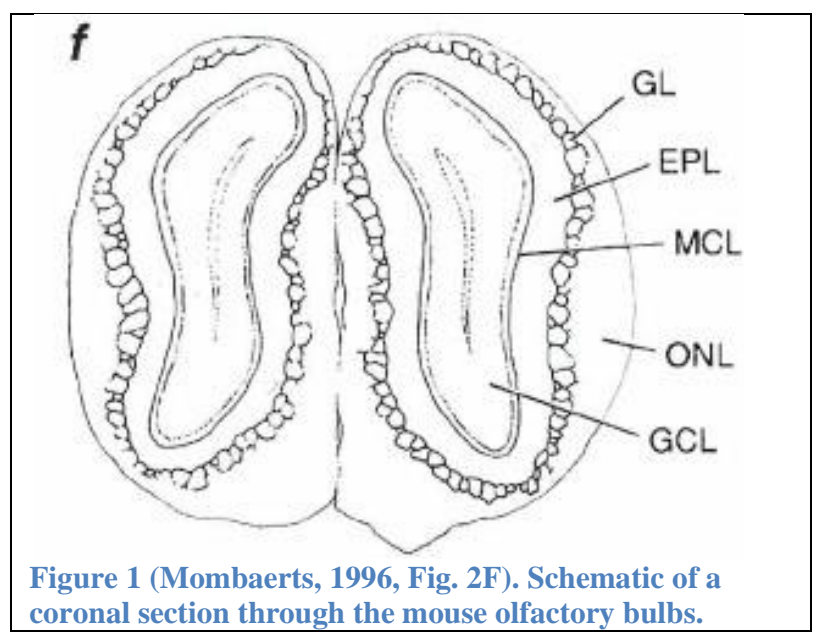

There exists debate as to which cells are encompassed by the term "OECs". Some propose that, based upon morphological or antigenic variations, certain populations of these cells - particularly in the bulb - should be regarded as distinct subtypes. Lineage relationships are a tractable matter, but there is an inherent difficulty in defining cellular categories based upon features that may or may not be plastic. Thus, for the purpose of the following structural description, the broadest definition of OECs will be used, that is: those cells that ensheath the olfactory axons from the epithelium to the glomeruli.

The manner of this ensheathment is characteristic of OECs. The classic description is of a cell with a peripheral, irregular nucleus and cytoplasmic processes that form a "tube" around a large number of axons. These processes also branch internally to incompletely divide the channel into compartments with multiple axons per mesaxon (Fraher, 1982). The arrangement of axons in direct contact along their longitudinal extent is unique in the adult nervous system; it is only seen elsewhere during embryonic development (Gasser, 1956). The maintenance of an embryonic-like arrangement of axons is one of the reasons for which OECs were initially considered as important to the regenerative capacity of the olfactory system. 


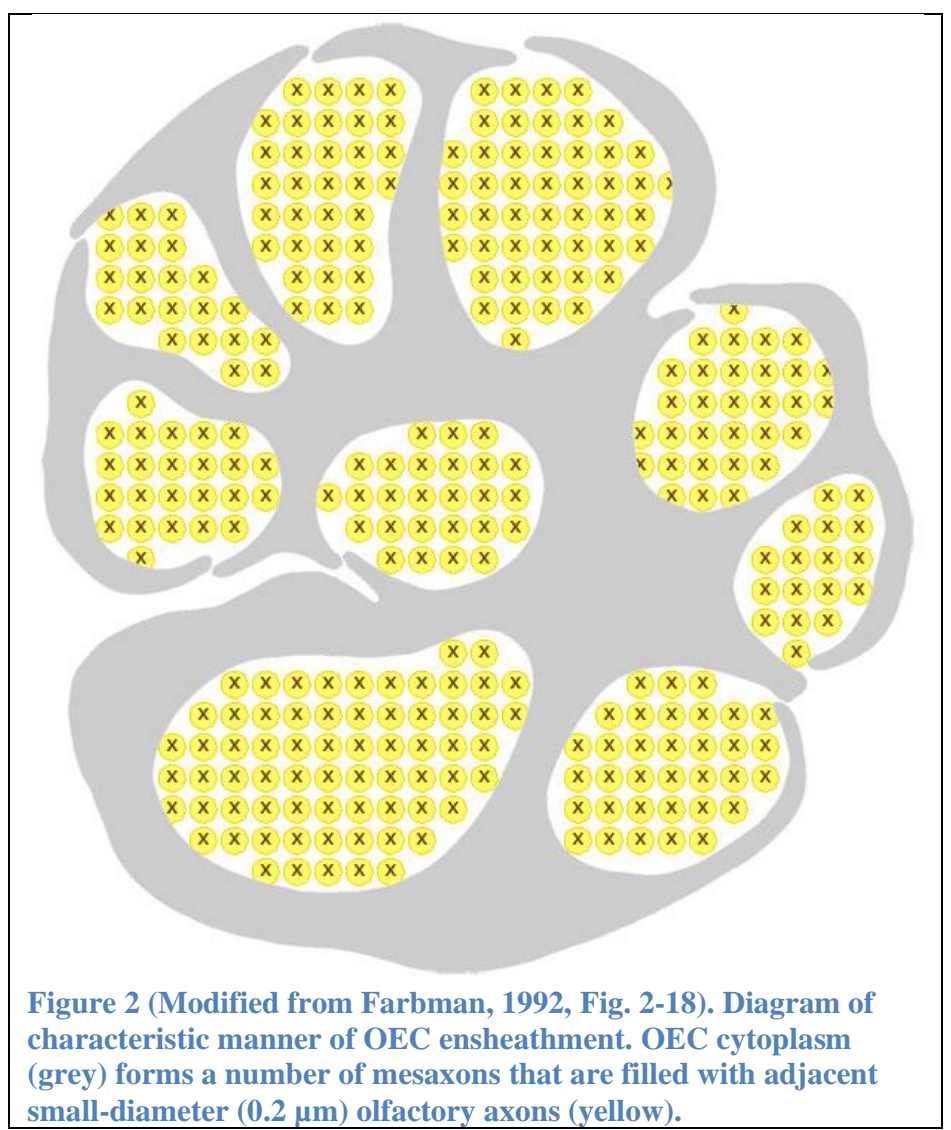




\section{2 - Epithelium}

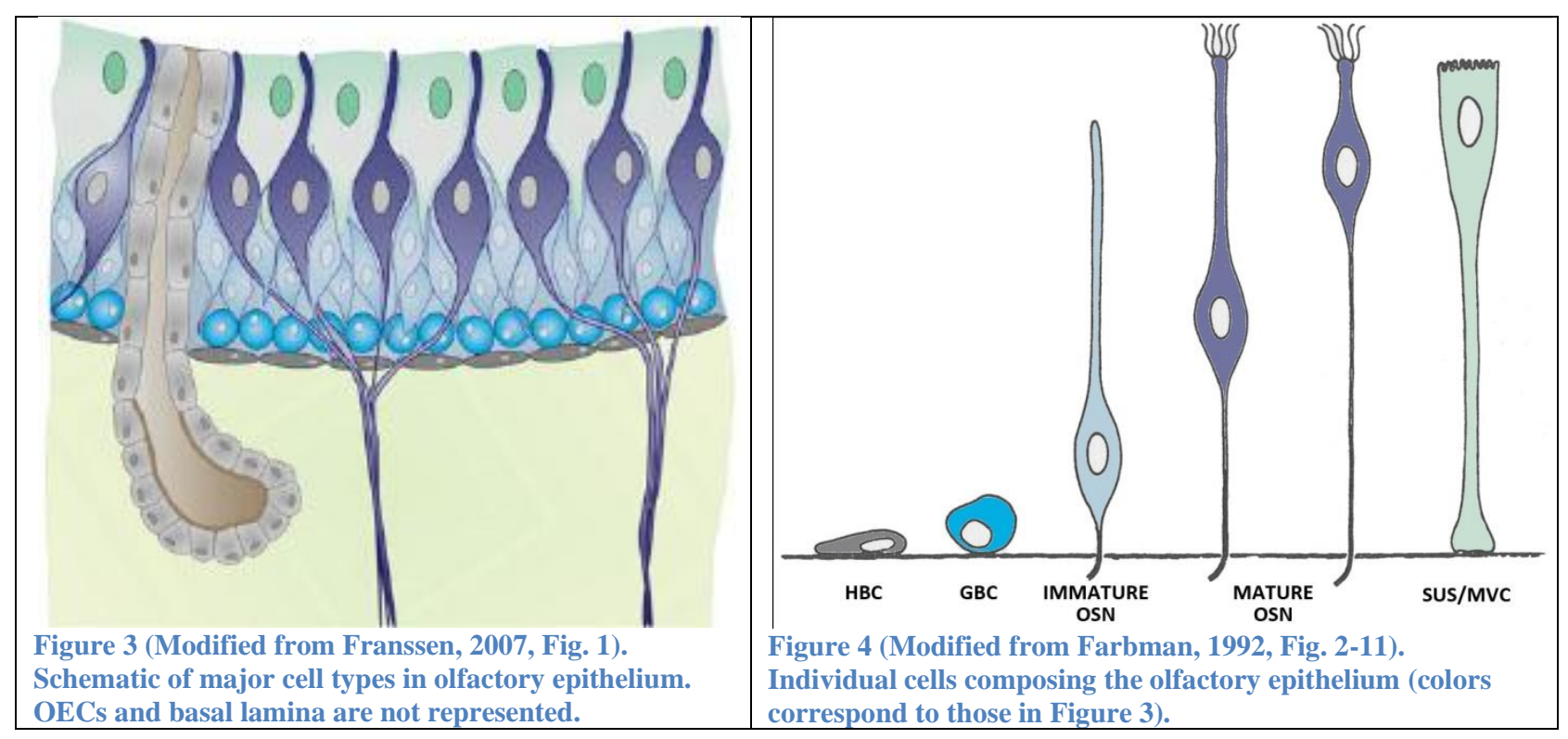

The olfactory epithelium is a pseudostratified epithelium with cellular somata organized into three general compartments. The perikarya of sustentacular and microvillar cells occupy the most apical compartment. These cells serve to phagocytose debris (Suzuki, 1996), maintain extracellular ionic concentrations, and isolate OSN dendrites from one another. The middle compartment consists of mature OSNs underlain by a population of immature OSNs. The process of maturation involves a gradual ascent of the OSN somata. Only fully mature OSNs possess the ciliated dendrites that extend to the apical surface (Figure 4). The basal compartment contains globose basal cells (GBC) and horizontal basal cells (HBC) that lie directly upon the epithelial basal lamina (reviewed in Farbman, 1992). The ducts of Bowman's glands traverse these compartments to convey the gland's secretions from the underlying lamina propria to the apical surface of the epithelium (Figure 3; Cuschieri \& Bannister, 1975b).

The axons of neighboring OSNs coalesce into small bundles as they descend towards the basal lamina (Figure 34; Cuschieri \& Bannister, 1975b). Once the basal lamina is reached, these axons turn and travel between the HBCs and the basal lamina to a greater or lesser extent before 
turning again to exit the epithelium (Figure 5). This was noted early-on by Gasser (1956),

"Another point, revealed by the Golgi prints, is the probability that the axon will cross the basement membrane, in terms of electron microscope dimensions, at enormous distances from orthogonal projections of cell bodies." The HBCs form spaced hemidesmosomes that tether them to the basal lamina and serve as anchors for the "HBC arches" that cover the underlying bundles of axons (Figure 6, Figure 7, Figure 8).

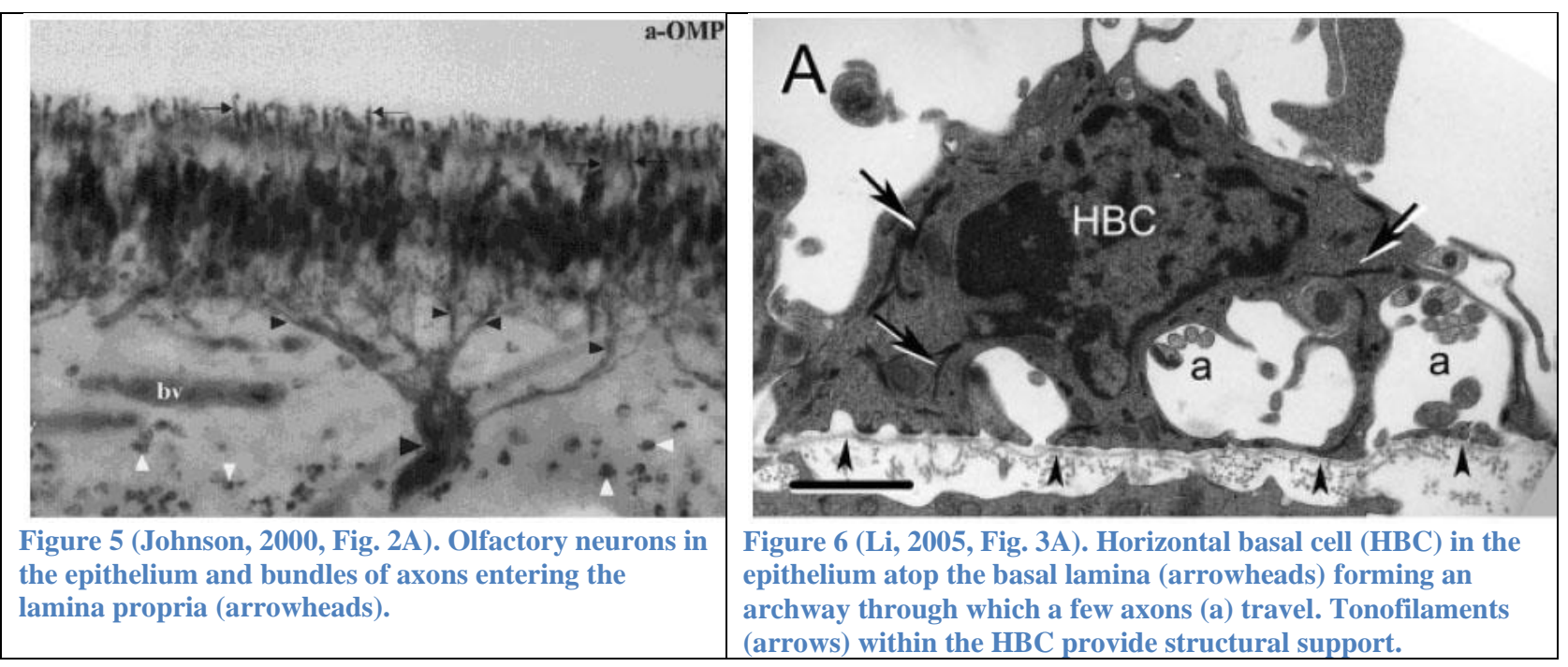

Occasionally, OEC processes (Figure 7) and perikarya (Figure 8) are found superficial to the basal lamina under HBC-formed archways where they ensheath small bundles of axons (Holbrook , 1995; Li, 2005; Herrera, 2005; Astic, 1998; Tennent \& Chuah, 1996). These OECensheathed olfactory bundles (EOB) are most typically observed near where the axons exit to the lamina propria. Thus, OECs are present but not abundant within the epithelium (Figure 9). It is noteworthy, however, that these intraepithelial OECs lack the external basal lamina (Figure 7, Figure 8) that is found along the main traverse of the olfactory fascicles (Figure 17) before it is shed in the ONL. 


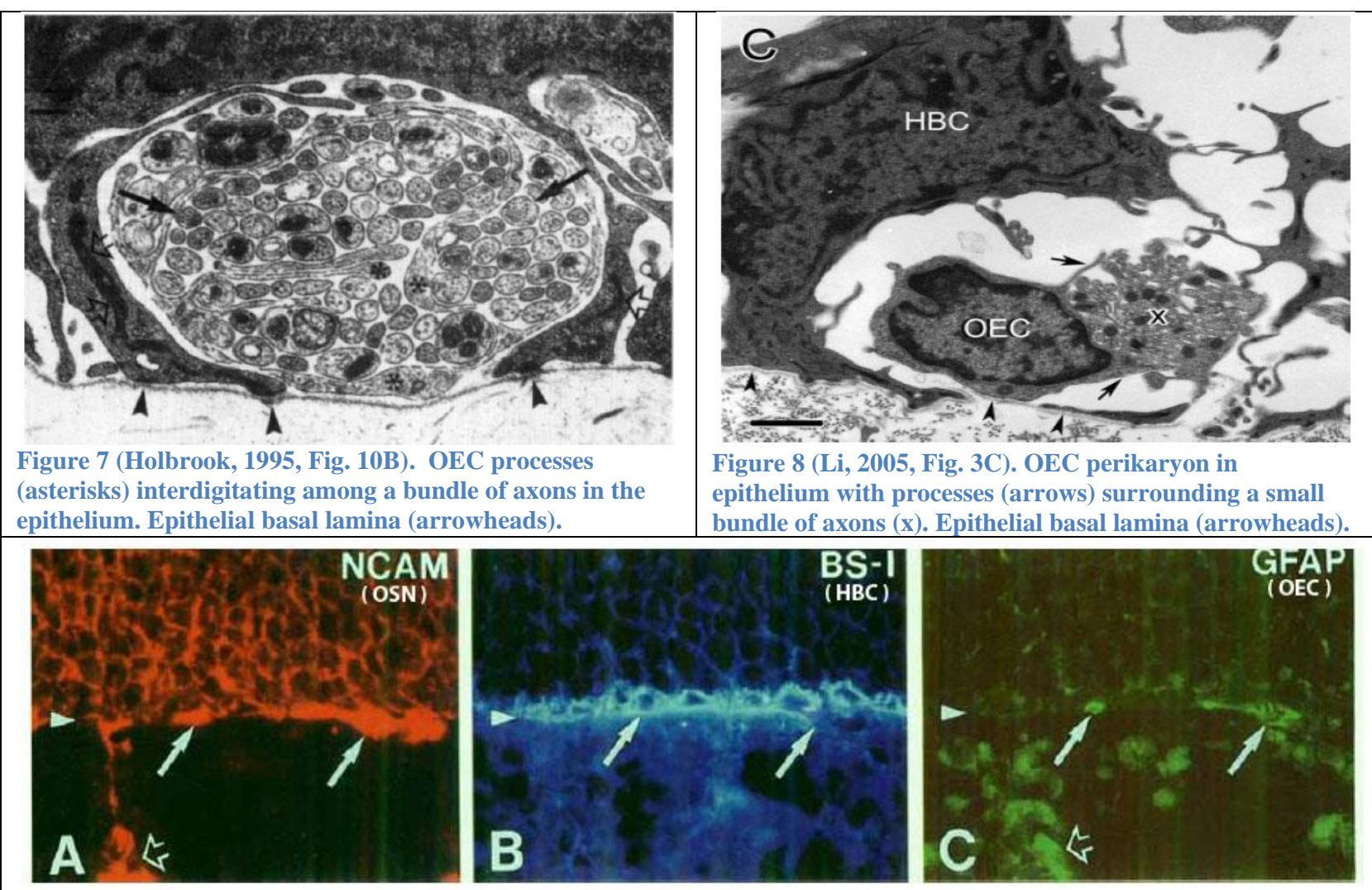

Figure 9 (Modified from Holbrook, 1995, Fig. 8). Triple immunohistochemical view of the olfactory epithelium. Arrows indicate the same area in each figure. Arrowhead indicates the epithelial basal lamina. Note that most of the HBC profiles (blue) are filled with axons (red), but fewer contain OEC processes (green).

In other instances, HBCs extend processes into the lamina propria in order to "hand off" bundles of axons to the underlying OECs (Herrera, 2005). This transitional region is thus a flexible one where OEC or HBC processes may extend into either compartment to ensure ensheathment of the axons.

The proximal spatial association of OECs and axons within the epithelium corroborates data from developmental studies that show an equally early temporal association. The first axons lead as they breach the epithelial basal lamina, and are then followed by migratory cells (Farbman \& Squinto, 1985; Marin-Padilla \& Amieva, 1989). Some of these migrating cells are considered to be OEC progenitors (pOEC) since they migrate with - although lagging - the growing axons, and neighbor them along their length. This relationship later develops into the 
characteristic manner of OEC ensheathment (Fraher, 1982; Cuschieri \& Bannister, 1975b; Doucette, 1989; Valverde, 1992).

In certain histological sections, some of these migrating cells have been observed with a “trailing tail” still in the epithelium (Farbman \& Squinto, 1985; Marin-Padilla \& Amieva, 1989). It is possible that these cells are the pOECs who, by way of maintained association with the axons, develop into the adult OECs observed within the epithelium. This might explain the colocalization of intraepithelial OECs to regions near axon exit, as well as their relative sparseness.

Though it is possible that OECs from the lamina propria encroach into the HBC archways, there are two reasons why the above hypothesis of an early intraepithelial association is more attractive. First, it does not seem necessary for OECs to extend in a manner retrograde to the direction of axonal growth to accomplish an ensheathment that HBCs capably achieve - as demonstrated by those HBC processes that descend into the lamina propria to deliver the axons to OECs (Herrera, 2005). Second, it has been demonstrated that OECs are derived from the olfactory placode which forms the olfactory epithelium (Couly \& Le Douarin, 1985; MarinPadilla \& Amieva, 1989; Chuah \& Au, 1991; Norgren, 1992). Given that OECs maintain their association with the axons at all stages, it is not unreasonable to assume that this began in some cases while still within the epithelium. 


\section{1 - What pioneers the pathway: OECs, olfactory axons, or both?}

This question of what pioneers is relevant to efforts to use OECs to foster regeneration because OECs are well-established promoters of axonal growth, and understanding how to control their engraftment and migration will enhance their therapeutic potential. If OECs pioneer the olfactory pathway ahead of axons, the migratory cues employed during development could be studied and exploited for therapeutic purposes. For example, the implications are clear-cut if OECs migrate towards the bulb in response to specific factors, and the axons grow towards the bulb simply due to the presence of OECs as a substrate. In this case, regeneration could utilize the same mechanisms, and any tropic role in axon growth would be minimized. Under these circumstances, it would more likely be the case that OECs transplanted into a lesioned spinal cord, for instance, would promote the functional regrowth of these neurons as well.

The observations of embryonic olfactory epithelium demonstrate that the axons are the first element to breach the epithelial basal lamina (Farbman \& Squinto, 1985; Marin-Padilla \& Amieva, 1989; Cuschieri \& Bannister, 1975b). When these axons reach the developing telencephalic vesicle, they again breach the early glia limitans - another basal laminar structure to the exclusion of any other cell type (Doucette, 1989; Valverde, 1992; De Carlos, 1995). So on structural grounds, it is likely the axons have a key role in the progress of olfactory nerve development.

The opposite conclusion was drawn from a study of newborn rat, newborn opossums and 64-day-old opossum tissue (Tennent \& Chuah, 1996). Here it was reported that the processes of OECs clearly extended further toward the telencephalon than the axon tips, leading their growth out of the epithelium and through the lamina propria (Figure 10). The study routinely substituted spatial relationships in the histological sections for temporal ones (see caption, Figure 10). They 
describe a section containing a nerve bundle near the epithelium as having recently emerged from it, despite the fact that only a single section of a 64-day-old opossum is described. Though this type of temporal metaphor is conventional when describing spatial pathways, their subsequent discussion reveals it as a conflation used to lend temporality to the observation of OEC processes extending ahead of the axon terminals. This observation itself is not convincing and may be artefactual as the axon bundle pictured exhibits a number of orientations along its length, and may have simply exited the plane of section rather than being at its terminus. Without a serially-sectioned demonstration of the distal limit of the axons, the whole premise collapses, and renders the data inadequate to support the idea that OECs lead the growth of olfactory axons to the bulb.

Numerous in vitro assays have demonstrated that axons preferentially grow on a substrate of OECs (Tisay \& Key, 1999; Chuah \& Au, 1994; Goodman, 1993). Some have taken this as support for the aforementioned in vivo observation to conclude that OECs pioneer axon growth in the olfactory pathway (Tisay \& Key, 1999). It should not be surprising that OECs promote axon growth as this is one of their primary functions in established olfactory nerves: to support the extension of axons by immature OSNs. But this cannot be taken as implicit support of the idea that OECs pioneer their embryonic development. The earliest source tissue utilized in these in vitro assays was rat E19.5 (Tisay \& Key, 1999), which is much later than the time points during which the first axons leave the epithelium -around E10-11 in the mouse (Cuschieri \& Bannister, 1975b; Doucette, 1989), and E12-12.5 in the rat (Valverde, 1992; Treloar, 1996). The early embryonic mesenchyme is rich with axon growth-promoting matrix molecules that diminish postnatally (Julliard, 1998). So the earlier tissues are highly permissive on their own, 
while the late embryonic to postnatal tissues present a different set of cellular conditions than those present during the ontogeny of the pathway.

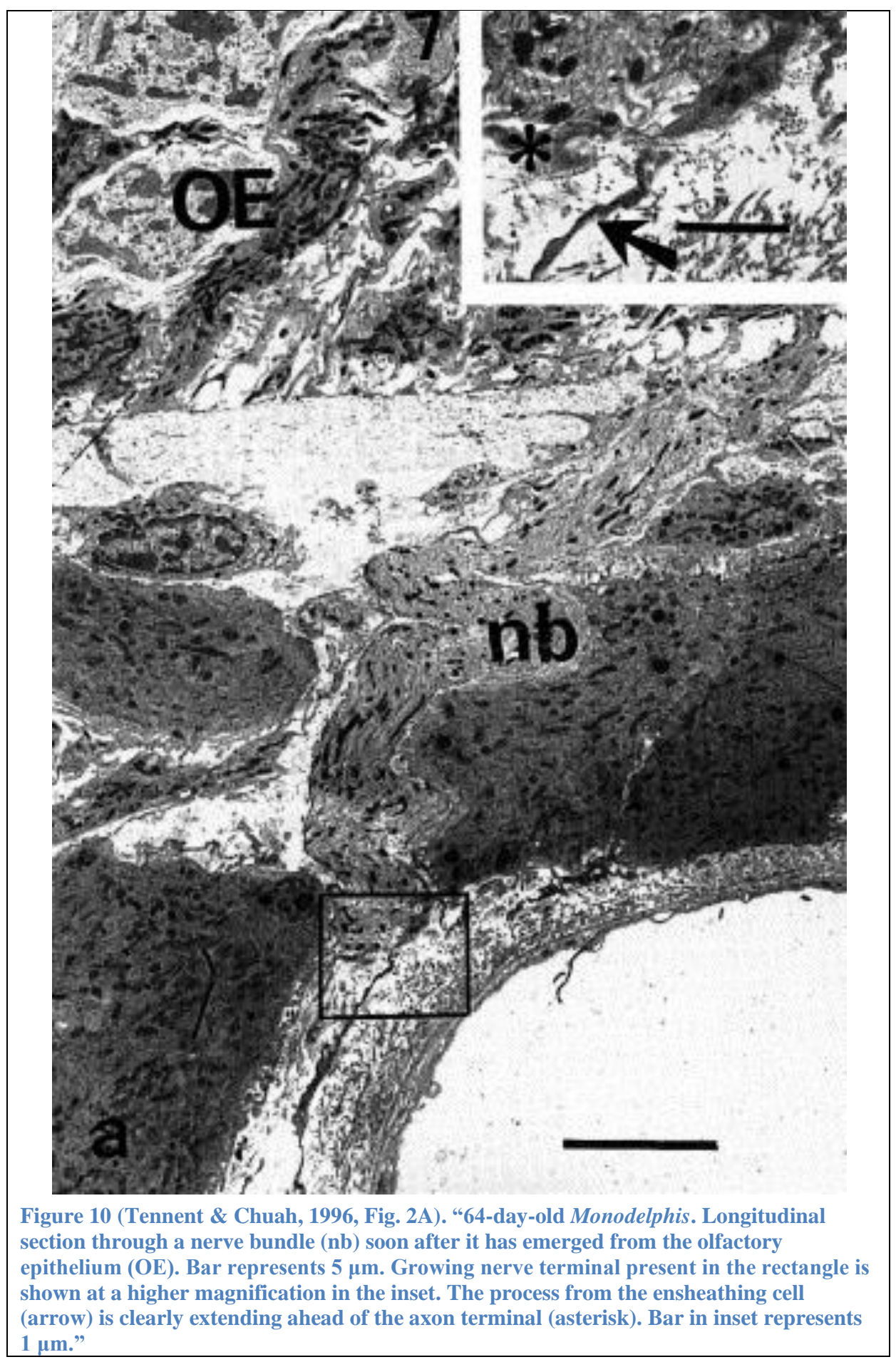


Along the same lines, even if Tennent \& Chuah's (1996) study is valid and reflects OECs leading the growth of axons it, too, was limited to postnatal animals. During embryonic development the OECs at first are loosely associated with the axons and only gradually begin to ensheath them (Fraher, 1982; Gong, 1994; Cuschieri \& Bannister, 1975b), before producing basal lamina at a later stage. In the rat, this basal lamina appears just before birth (Fraher, 1982; Julliard, 1998). Observations of OECs situated distally in the developing pathway of the mouse show a similarly timed appearance of the basal lamina that forms the new glia limitans of the bulb (Doucette, 1989). Thus, during their early embryonic growth the axons and OECs are exposed to the broader extracellular environment, but their exposure ends at a specific time. This change is quite likely reflected in the manner in which new fascicles would grow after this period. This is one reason that observations of postnatal fascicle growth may not be relevant to their ontogeny.

Another study oft-cited as support for the hypothesis that OECs pioneer the olfactory pathway claims that soluble factors from the bulb attract OECs (Liu, 1995). In other words, factors ostensibly emanate from the developing bulb and attract a "carpet" of OECs from the epithelium upon which the axons then extend. The evidence for this interaction came from in vitro experiments in which bulb or cerebral explants are placed on a monolayer of purified, DiIlabeled OECs. In this setting, the OECs migrated only towards the bulb explant (Figure 11).

For purposes of studying the initial establishment of the olfactory pathway, the methods employed were suboptimal. First, explants were obtained from P1-3 rats. At this stage, the bulb has already developed and been contacted by a robust population of axons and OECs. To make any claims about primary development, it would have been necessary to utilize the portion of the telencephalon that develops into the bulb before it has been contacted by any axons or OECs. In 
preparing the bulb explant, they manually removed the ONL, which is rich with OECs, and claim that the DiI-labeled OECs are not migrating towards residual OECs. However, use of $\mathrm{p} 75^{\mathrm{NTR}}$ as a marker for OECs demonstrates that a small number of them remain despite removal of the ONL.

Since $\mathrm{p} 75^{\mathrm{NTR}}$ is only expressed by OECs on the outermost layers of the ONL (Au, 2002; Franceschini, 1996; Astic, 1998; Treloar, 1999), it is highly likely that a greater number of OECs remained within the explant. Moreover, even if all of the OECs had been removed, the findings cannot preclude the possibility that the explant itself was conditioned by its prior contact with OECs and axons. For the same reason, an OEC chemotaxis assay that this group performed utilizing bulb-conditioned medium is also inconclusive. In fact, recent studies have shown that OEC proximity and OEC-secreted factors such as GDNF do indeed increase OEC mobility (Windus, 2007; Cao, 2006). In conclusion, in order to study whether soluble factors from the bulb could be responsible for the primary development of the olfactory nerves, it would be necessary to utilize the region of the telencephalon that will develop into the bulb before it has been populated by OECs. 

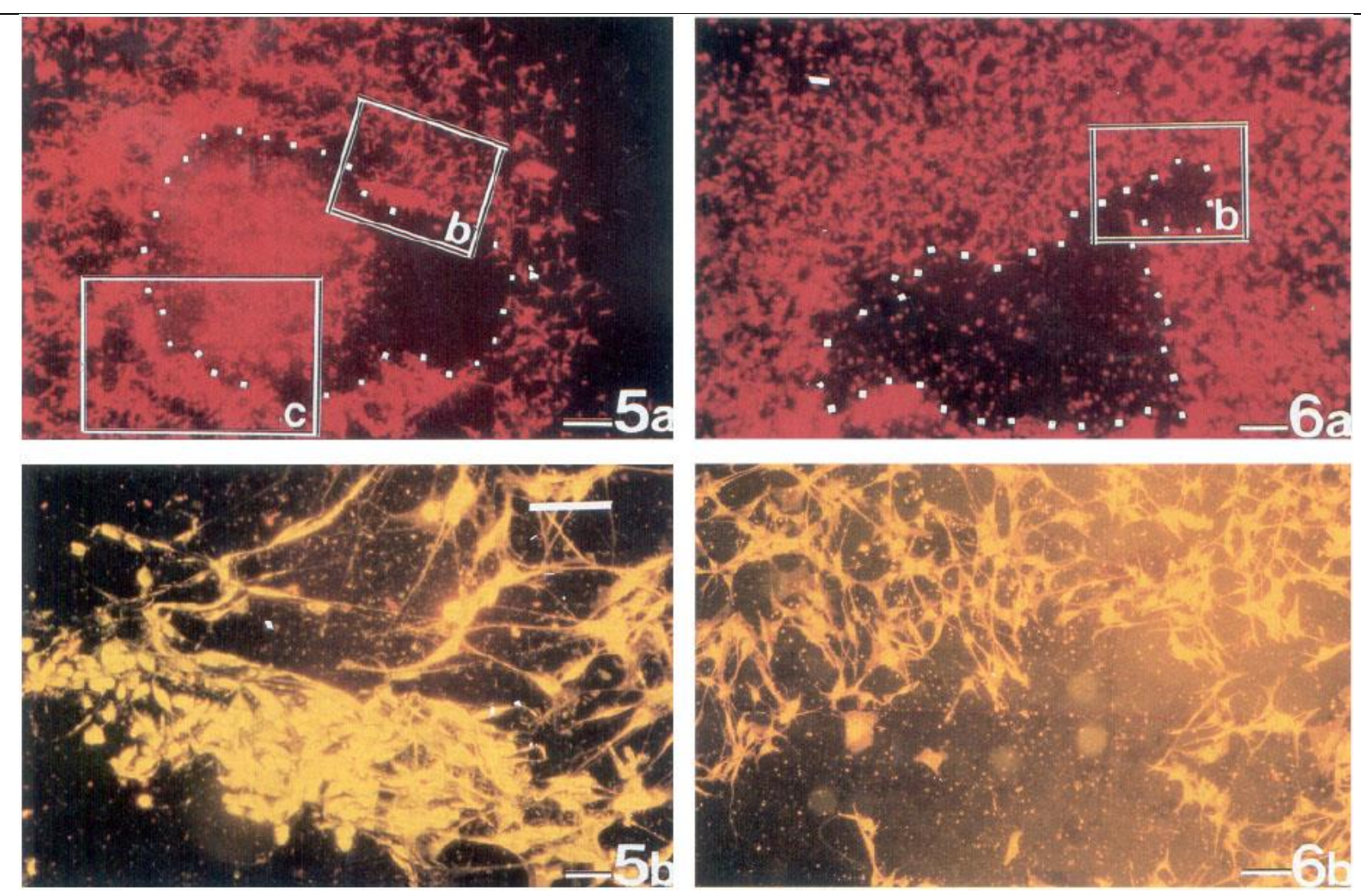

Figure 11 (Liu, 1995, Figs. 5AB, 6AB). DiI-labeled OECs after coculture with bulb explant (left panels) or cerebral explant (right panels). The dotted lines indicate the position of the explants before they were removed. OEC migration toward the bulb is suggested by the close layering of labeled OECs at the border of the explant, followed by a region of lower OEC density.

In fact, the observation that the DiI-labeled OECs do not migrate towards cerebral explants may contradict their conclusion, and provide more weight to the concept that axons pioneer the olfactory pathway. Various bulbectomy and olfactory placode transplant experiments have demonstrated that the olfactory epithelium is capable of projecting axons to form protoglomerular and ONL-like structures in ectopic or even artificial substrates (Graziadei, 1978, 1979; Graziadei \& Monti-Graziadei, 1986; St. John, 2003; Storan \& Key, 2004; Chehrehasa, 2006). The pathway is able to form without any factors unique to the presumptive bulb. All such ectopic regions could be considered as equivalent to the cerebral explant used in this study, and since it demonstrated that OECs did not migrate towards the cerebral explant, it is probable that 
axons would be entirely responsible for pathfinding, at least within the context of the in vitro explant setting.

Finally, in vitro results from Storan \& Key (2004) suggest that axon tropism is more responsible for laying down the pathway to the bulb. They found that axons entered bulb and cerebral, but not tectal, tissues, while OECs freely migrated across all three. The result that the OECs have no pronounced tropic response makes it unlikely that they are specifically responsive to the anlage of the bulb. As a consequence, it seems that the OECs are more likely to be following along behind the growth cones of axons. They clearly do migrate towards postnatal bulb explants in vitro, whether toward other OECs within the explant or some other factors, but they have not been observed migrating in the postnatal mesenchyme in the absence of axons, nor have they been convincingly observed migrating just ahead of axons. It may be worthwhile to compare the two likely and verified sources of OEC tropism - olfactory axon growth cones and postnatal bulb - to see if there are any contact or diffusible factors that overlap.

Taken together, the available observations point to a tropic role for the axons and a trophic role for the OECs during ontogeny; the axons pathfind and "pioneer", while the OECs migrate alongside and support their growth. It remains possible that the cellular environment in postnatal animals is such that OECs must completely enclose bundles of axons, and as such could appear to lead their growth. At this stage, however, the bulb has already developed and, as Liu (1995) demonstrated, contains some source of tropic factors which influence OECs. Since even in this scenario, OECs have not been observed migrating towards the bulb far ahead or in the absence of axons, it may be that during postnatal axon growth the OECs must isolate the growing front of axons, but it is still the axons that are required for extension or pathfinding. 


\section{2 - Beyond the Epithelium}

After the small bundles of axons exit the epithelium, they continue to merge into larger bundles in a spatially-defined manner (Chehrehasa, 2006; Storan \& Key, 2004; Mombaerts, 1996; Au, 2002; Julliard, 1998). Thus, from the large convoluted sheet of the olfactory epithelium, thousands of axons eventually merge into the tens to hundreds of large bundles that pass through the foramina of the cribriform plate. This set of large bundles will eventually merge into a singular axonal plexus in the ONL. While some of this merging begins in the epithelium with adjacent axonal processes coalescing as they descend basally (Figure 5, Figure 34) and course through the HBC tunnels (Figure 6, Figure 7, Figure 8) - the majority of it occurs within the lamina propria.

The lamina propria is an area of considerable interaction between OECs and olfactory axons. The differences in structure of the olfactory fascicles throughout the depth of the lamina propria are best understood by bearing in mind the ongoing addition of axons with time and their increasing association with distance from the epithelium as well as their growth in advance of the OECs during development. 


\section{3 - Lamina Propria}

In the lamina propria, the manner of OEC ensheathment is similar to that seen in the epithelium, but here, no axons are left unensheathed by OECs. Every bundle of axons exiting the epithelium is completely surrounded by the cytoplasm of one or more OECs to form an EOB.

Throughout the depth of the lamina propria, the outside of each EOB is apposed to and surrounded by a continuous basal lamina (Figure 12), and is thus called a laminated EOB (LEOB). This basal lamina is continuous with the epithelial basal lamina at the points of axon exit from the epithelium, as well as the glia limitans at the surface of the bulb (Herrera, 2005; Doucette, 1990; Field, 2003; Raisman, 1985; Barber \& Lindsay, 1982). Thus, OECs constitute a channel through the lamina propria that connects the epithelium with the bulb. This channel makes the two regions continuous, delineated by an unbroken border of type IV collagen, laminin, and fibronectin (Figure 13; Julliard, 1998).

Elsewhere in the literature, the arrangement of axons, OECs, and adherent basal lamina is sometimes referred to as a fascicle. Inconsistent usage of the terms bundle, fascicle, fiber, and nerve, has complicated translation of anatomical details across primary sources. In keeping with the usage for other nerves and skeletal muscle, the term "fascicle" will be reserved for the anatomical unit that includes the connective tissue. This will help clarify the discussion of the collagenous and olfactory nerve fibroblast (ONF) investment around and upon LEOBs. However, for better or for worse, the verb "fasciculate" will continue to be used conventionally that is, the longitudinal merging of axons, irrespective of other surrounding or intervening elements. Thus, axons may fasciculate, but the resultant structure is not necessarily a fascicle. 


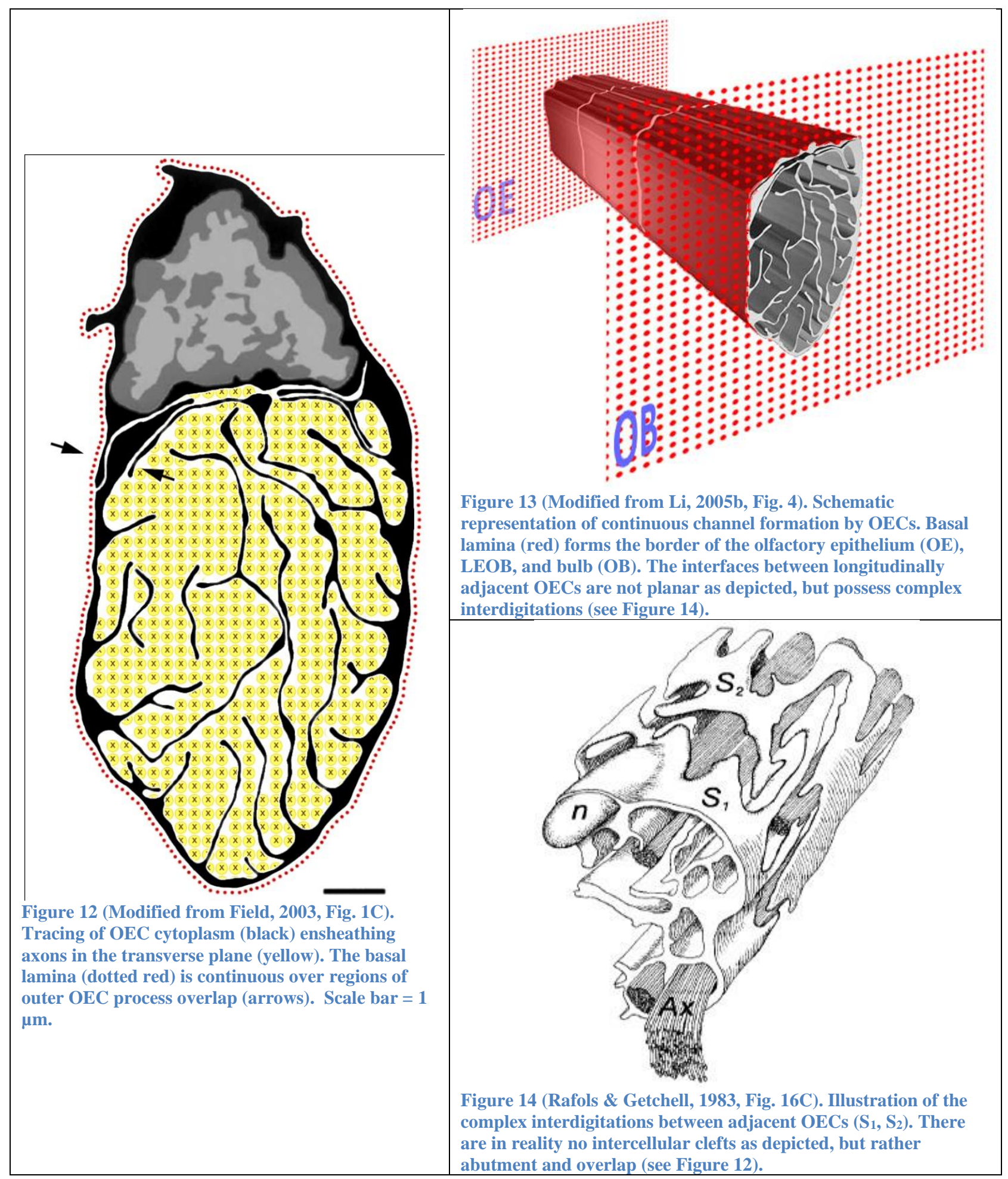


The LEOBs take over for the EOBs within the epithelium and are often a continuation of these. As a consequence, EOBs in the epithelium are usually comparable in size to the LEOBs found at the most superficial levels of the lamina propria (compare Figure 7 and Figure 15). A sense of the area from which the axon bundles take origin can be derived from the usual density of neurons in rat epithelium (5-10 x $10^{4}$ dendritic knobs per $\mathrm{mm}^{2}$; Farbman, 1992; Menco, 1980a; Hinds \& McNelly, 1981). For example, the rat LEOB in Figure 15 contains approximately 150 axons and measures $20 \mu \mathrm{m}^{2}$, and so represents the OSNs originating in approximately $2000 \mu \mathrm{m}^{2}$ of epithelium.

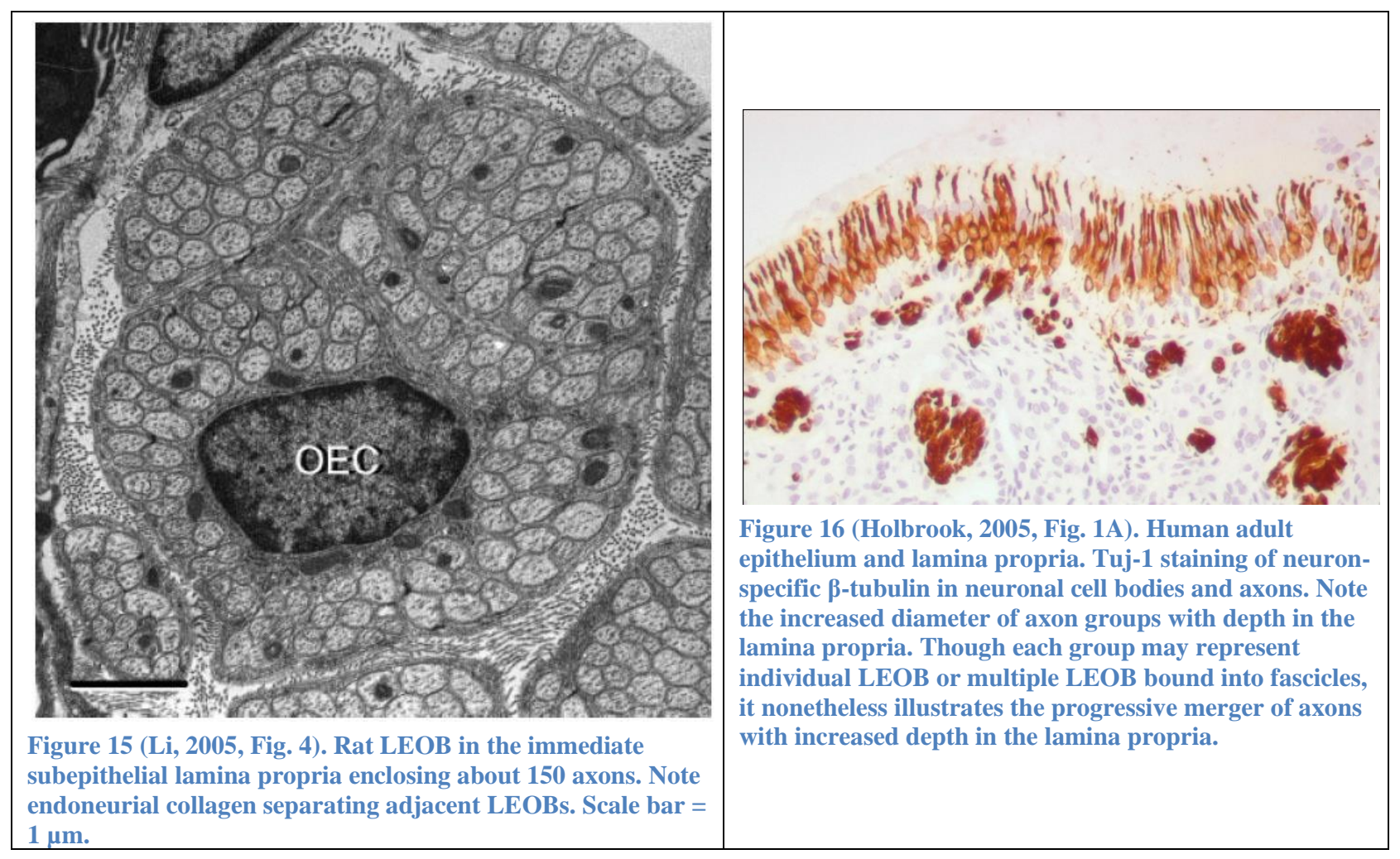




\section{1 - LEOB Merger}

The LEOBs increase in size as they descend deeper into the lamina propria, reflecting their continued convergence (Figure 15, Figure 17; Skinner, 2005; Doucette, 1990; Julliard, 1998). The convolutions of the epithelium lining the nasal turbinates leads to a range of LEOB sizes in a given plane of section, but overall the distribution of sizes increases with increasing depth (Figure 16; Herrera, 2005). Not only are more axons contained per LEOB, but the nuclei and cytoplasmic processes of multiple OECs can be identified contributing to the same LEOB in a given plane of section (Field, 2003; Fraher, 1982; Barber \& Lindsay, 1982; Kawaja, 2009). While remaining discrete cells, these cooperating OECs produce basal lamina only on the external surfaces of the LEOB as a whole (Figure 17). The spatial merger of axons is thus accompanied by this merger of OECs with their external basal laminae, resulting in a reduction in the number of discrete LEOBs as the bulb is approached (Julliard, 1998). 


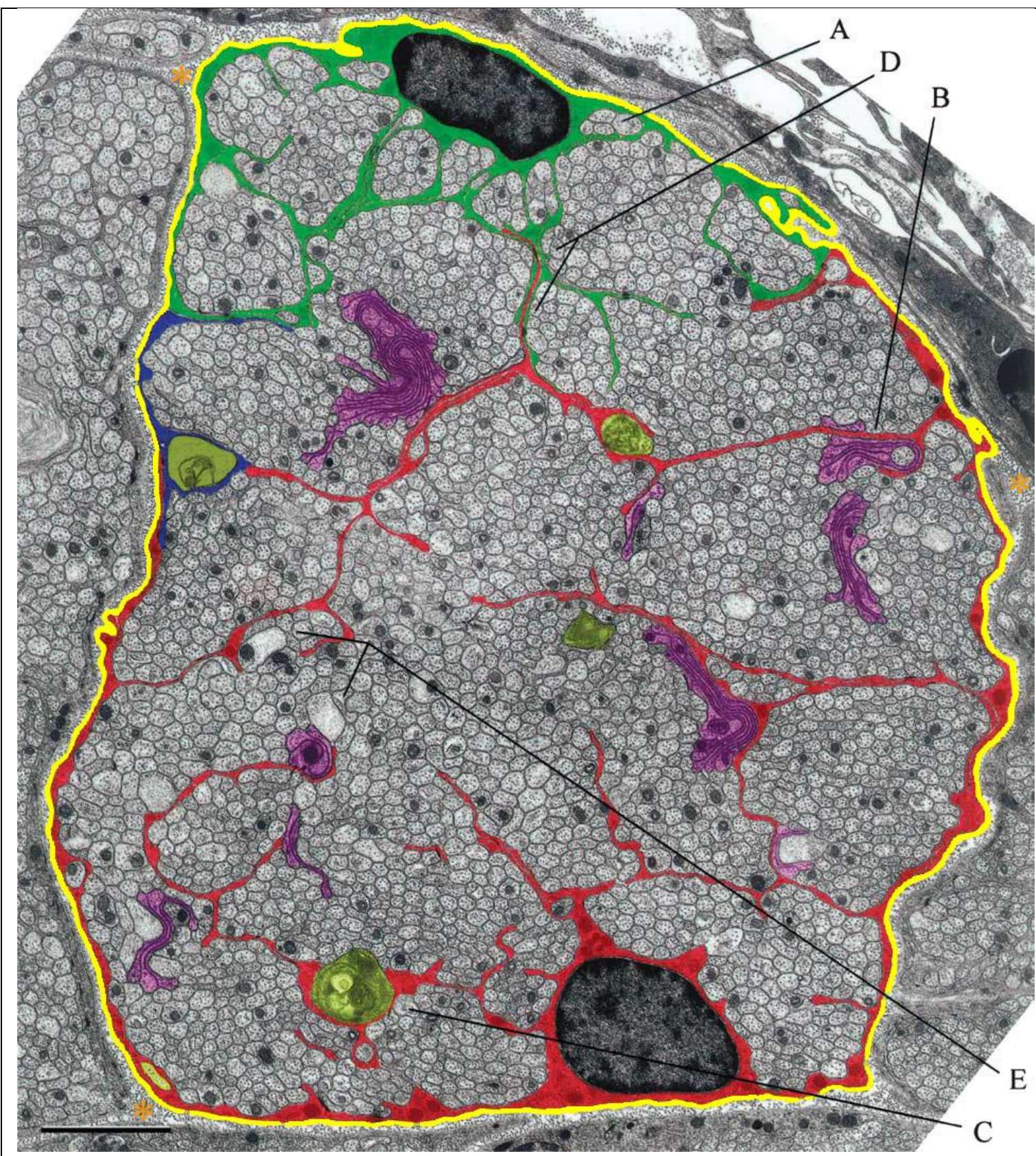

Figure 17 (Modified from Field, 2003, Fig. 2). Rat LEOB deeper in the lamina propria. The cytoplasm of three different OECs (red, blue, and dark green) cooperate to form complete ensheathment, and are surrounded by a continuous external basal lamina (yellow). There is endoneurial collagen (orange asterisks) between adjacent LEOBs. Scale bar $=2 \mu \mathrm{m}$. 


\section{2 - How would cooperative ensheathment arise?}

The manner in which the axons and pOEC converge en route from the olfactory placode towards the telencephalon during embryonic development provides a likely explanation for the variety of ensheathment morphologies observed along the length of the projection. In particular, cooperative ensheathment is more frequently observed in increasingly distal portions of the nerve. The accumulated data suggest that the flexibility with which early OECs ensheath all axons in their vicinity results in multiple OECs ensheathing a given bundle after smaller EOBs have converged to a greater degree. (It could be said that the morphology of the ONL, as we will see, is explained by this very fact.)

As previously mentioned, during embryonic development, the pOECs migrate with the axons extending towards the bulb. The pOECs actively divide along the axons' length in order to maintain their association where present, and to keep pace with the growing front of the axons as they advance toward the telencephalon (Cuschieri \& Bannister, 1975b; Marin-Padilla \& Amieva, 1989; Valverde, 1992). At the initial stages, the pOECs are loosely peripheral to the bundles of axons and do not possess the extensive cytoplasmic processes or basal lamina that ensheath and isolate them from the mesenchyme (Doucette, 1989; Farbman \& Squinto, 1985; Cuschieri \& Bannister, 1975b; Fraher, 1982; Marin-Padilla, 1989; Valverde, 1992).

Gradually, these transitional pOECs (p/OEC) begin to thread cytoplasmic processes around and through the bundles of axons. Fraher (1982) provides a highly detailed description of the establishment of this ensheathment. Each bundle may receive processes from multiple neighboring OECs, and each OEC may send processes to multiple bundles. Then:

"By 20 days, most vomeronasal nerve branches had become subdivided into a number of discrete axon bundles, each surrounded by a complete cytoplasmic sleeve. The sleeves of adjacent bundles had become separated from one another, over an increased proportion of their circumference, by basal lamina which was 
present as clumps of wispy material over parts of each bundle's circumference. Small amounts of interfascicular collagen were sometimes present in such areas. However, basal lamina remained absent over segments of the circumference of all bundles. Here, the sleeve processes of adjacent bundles were closely apposed to one another and occasionally passed from one sleeve to another." (Fraher, 1982, pg. 155)

Thus, there is a type of critical period before which a p/OEC gropes its way toward any axons in its vicinity. After this period, the ensheathment of individual axon bundles tightens, and a surrounding basal lamina appears. This is the point at which neighboring OECs contributing to the same bundle become incorporated within a single LEOB. Indeed, Fraher notes the occasional presence of multiple OEC nuclei within certain LEOB sections at this stage. The exact timing of this stage cannot necessarily be extrapolated from the vomeronasal nerve to the main olfactory nerves, only that its manner of development is likely similar. The emergence of mature basal lamina around the main olfactory nerves in rats is complete at E17-E18 (Julliard, 1998).

The reason that cooperative ensheathment is more pronounced at points further along the course of the olfactory nerves towards the bulb is the result of spatial merger during this early period. The early axon bundles exit from the epithelium accompanied by a contingent of pOECs from the same area. Bundles merge as they approach the bulb - the axons converge, and so do the pOECs that accompany them. We can consider the axons and their accompanying pOECs as a kind of migratory unit. Compared to the vomeronasal projection, the main olfactory projection in mammals has a larger epithelial area and is relatively closer to the bulb, so many of these migratory units will not converge until they come into the immediate vicinity of the bulb. There is thus an inherent separation between many of the olfactory migratory units at superficial depths that is not as pronounced in the vomeronasal nerve - which has a smaller sensory surface and a more distant target. 
While the proximity of the vomeronasal migratory units is perhaps what occasioned the level of detail provided by Fraher (1982) ${ }^{1}$, it is consistent with the transition of migratory pOECs to ensheathing OECs in the olfactory nerves as well (Doucette, 1989; Farbman \& Squinto, 1985; Cuschieri, 1975b; Marin-Padilla, 1989; Valverde, 1992). This mature ensheathing relationship among p/OECs and axons emerges gradually and is completed after the contact of early axons with the telencephalon. The appearance of basal lamina around OECs is a particularly late event in this progression. Substantial bundles form by merging in the deeper part of the lamina propria before the p/OECs develop basal lamina and group the axons into mature LEOBs. Thus, the cooperative ensheathment observed in many LEOBs, particularly the larger ones, is a result of merger of pOEC within separate migratory units prior to their maturation and establishment of basal lamina.

This is a hypothesis to explain the unique manner of ensheathment observed in many olfactory fascicles. It utilizes limited spatial and temporal observations in an attempt to understand the variety of extant formations. It may or may not be causally true. It is nevertheless natural, when sketching out a physical history, to impart some sort of narrative hypothesis. This not only helps to structure the facts, but provides something that may be tested. 


\section{3 - Olfactory Nerve Fibroblasts}

One or more LEOBs are cordoned into fascicles by thin encircling layers of perineurial olfactory nerve fibroblasts (ONF) that express basal lamina on various surfaces. The degree of perineurial encirclement appears to vary between species: mice and rats have a few thin layers (Figure 17, Figure 18; Kawaja, 2009; Field, 2003; Li, 2005; Schwob, 1992; Fraher, 1982), while cats, humans, and macaques have a greater number (Figure 19, Figure 25, Figure 30; Kawaja, 2009; Smithson 2009; Holbrook, 2005; Herrera, 2005). In a detailed study of the macaque olfactory nerves, Herrera (2005) observed an increase in the number of perineurial ONF layers with progressive depth. It is possible that all species tend to exhibit the same progressive increase in ONF encirclement with depth, but that it is better observed in larger species due to the greater range in fascicle lengths.

Within these fascicles, the LEOBs are embedded in an endoneurial matrix of collagen (Figure 15, Figure 17, Figure 18, Figure 19; Doucette, 1991; Graziadei, 1980; Easton, 1971; Fraher, 1982). In the human and macaque there also exist prominent endoneurial ONFs that lack basal lamina and whose cytoplasmic processes intervene between LEOBs amidst the collagen bundles that already separate them within a fascicle (Figure 19, Figure 20; Holbrook, 2005; Herrera, 2005). Fraher (1982) noted the occasional presence of such fibroblasts in the endoneurial space of the rat vomeronasal fascicles. Such endoneurial ONFs may be present in most species, but the ubiquity of their processes in macaque and human olfactory fascicles is in marked contrast to the published observations of mouse and rat model systems. However, these observations too may be related to the depth of the fascicle and thus be more pronounced in larger species. 
In the absence of detailed data regarding the position of a fascicle in the lamina propria in other species, both of these generalizations about the primate olfactory nerves are tentative. If it is not merely fascicle size and depth that accounts for multi-layered perineurial ONF encirclement and the presence of endoneurial ONF processes, these inter-species differences may be the result of more complicated mechanisms - for instance, macrosmatic/microsmatic genetic differences - that may then be relevant to regenerative potential and model system generalizability.

One possibility may be explored by considering the human and macaque as equivalent both primates - but contrasting the pictured human (Figure 19) and macaque (Figure 20) fascicles. The former fascicle was obtained from an individual with chronic rhinosinusitis. This persistent insult to the olfactory epithelium causes degeneration of the axons, and a prolonged impairment of new OSN regeneration. Axonal degeneration typically results in OECs becoming hypertrophic as they phagocytose the cellular debris. This hypertrophy gradually resolves as the OECs degrade these debris ( $\mathrm{Li}, 2005 \mathrm{a})$. In this case, since the epithelium is chronically impaired in extending new axons into the LEOBs, resolution of the OEC hypertrophy may result in a general reduction in size of the LEOBs. If it is characteristic that primate fascicles are held open by multiple layers of perineurial ONF, it is possible that the endoneurial ONF cytoplasm must expand to fill the spatial void created by a collapse of the LEOBs. So, in a sense, the scanty endoneurial ONF cytoplasm in Figure 20 becomes the expanded cytoplasm of Figure 19. 


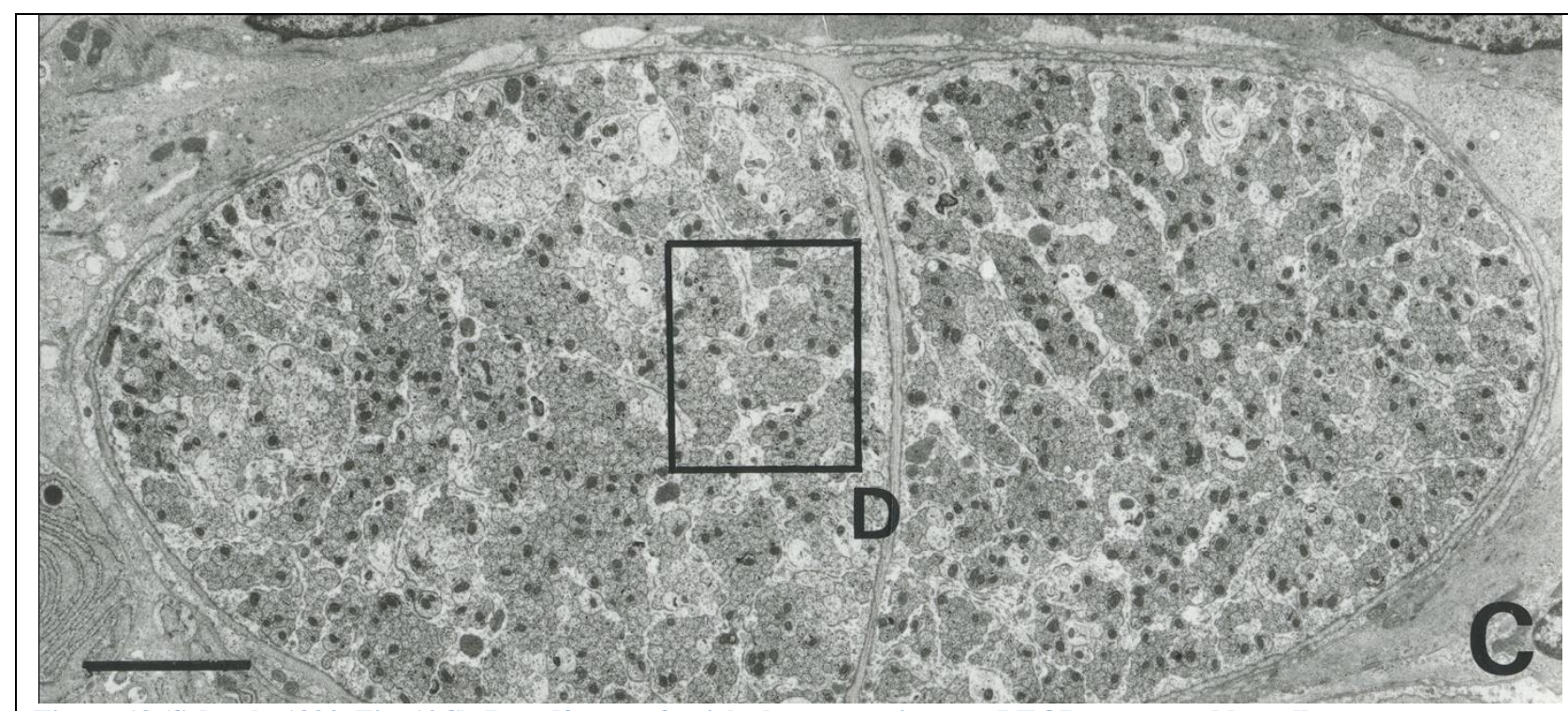

Figure 18 (Schwob, 1992, Fig. 10C). Rat olfactory fascicle demonstrating two LEOBs separated by collagenous endoneurium and surrounded by thin perineurial ONFs. Scale bar $=2.5 \mu \mathrm{m}$.

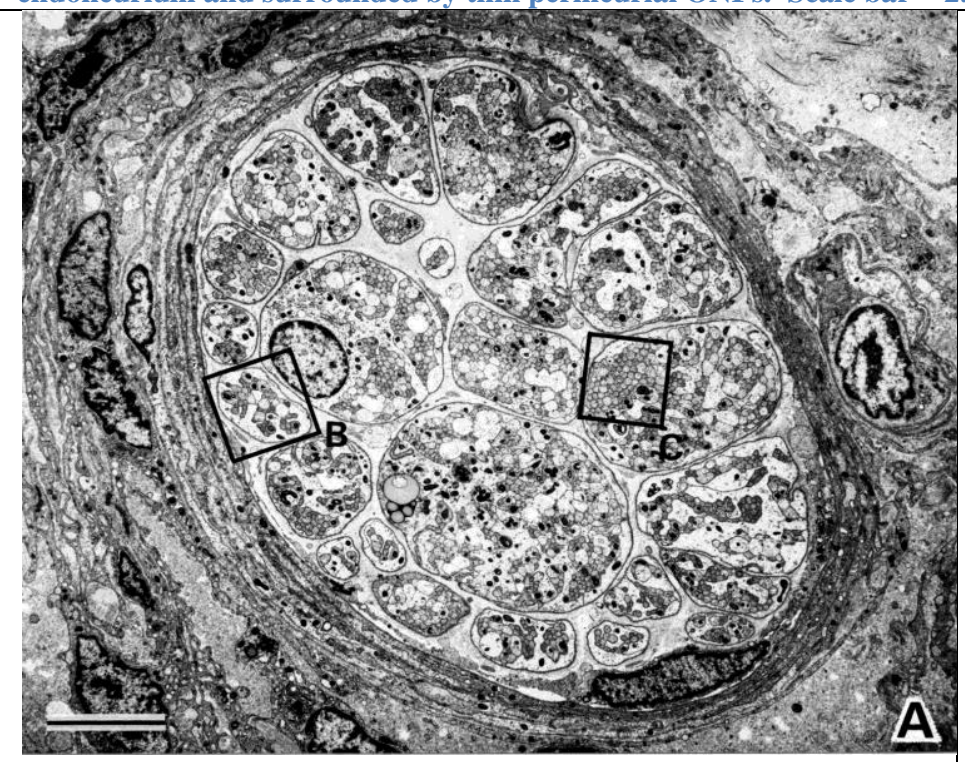

Figure 19 (Holbrook, 2005, Fig. 6A). Human olfactory fascicle demonstrating LEOBs, endoneurial ONF cytoplasm, and perineurial ONFs. The biopsy was obtained from a 58-year-old patient with chronic rhinosinusitis, so there is marked atrophy of axons within the LEOBs, possibly leading to the exaggeration of the endoneurial space. Scale bar $=5 \mu \mathrm{m}$.

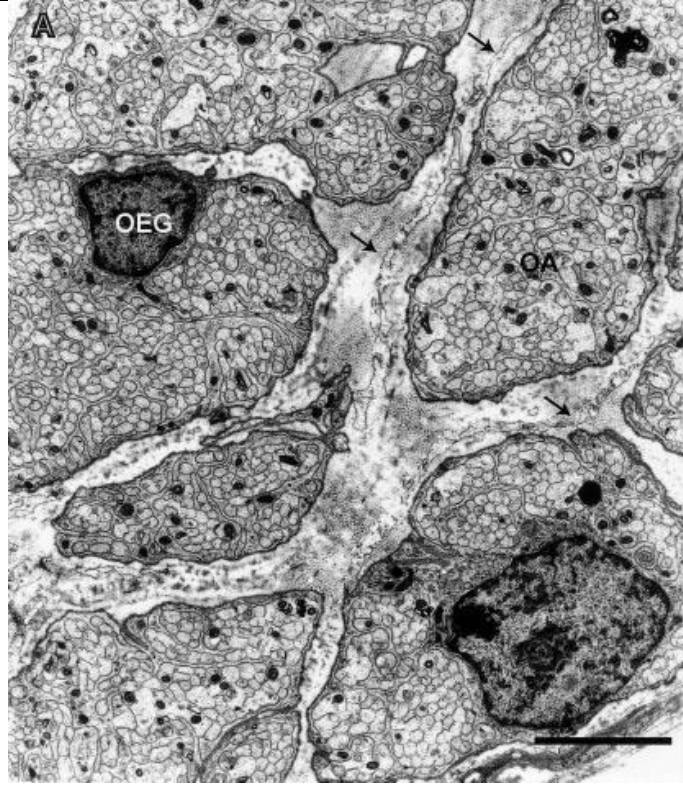

Figure 20 (Herrera, 2005, Fig. 4A). Macaque olfactory fascicle demonstrating $\mathrm{LEOBs}$, and thin slips of endoneurial ONF cytoplasm (arrows). Scale bar $=3 \mu \mathrm{m}$.

It was noted that in the human patient there was no evidence of aberrant collagen deposition, and their olfactory sensation eventually recovered after surgery that treated the underlying cause of the rhinosinusitis. Aberrant collagen deposition would likely have hindered the recovery of the pathway. It is possible that the endoneurial ONFs provide spatial flexibility in such circumstances to manage the vigorous patency provided by the multilayered perineurial 
ONFs. In this way, the increased layering of perineurial ONF in larger species may be associated with the more prominent appearance of endoneurial ONFs. In smaller animals (Figure 18), the lesser layering of perineurial ONFs may allow the whole fascicle to collapse somewhat in cases of chronic insult to the epithelium.

A related possibility is that the general absence of endoneurial ONF in mouse and rat (with the exception of Fraher's above infrequent observation) could be the result of bona fide macrosmatic/microsmatic differences and not just the result of limited perineurial ONF layering. It may be that olfactory neurogenesis and axon extension is much more active in such macrosmatic species and this, combined with their superior repertoire of functional odorant receptor genes, results in their fascicles being better "packed" with axons even under normal physiological circumstances. The endoneurial ONFs are thus spatially prevented from extending apparent processes in such animals.

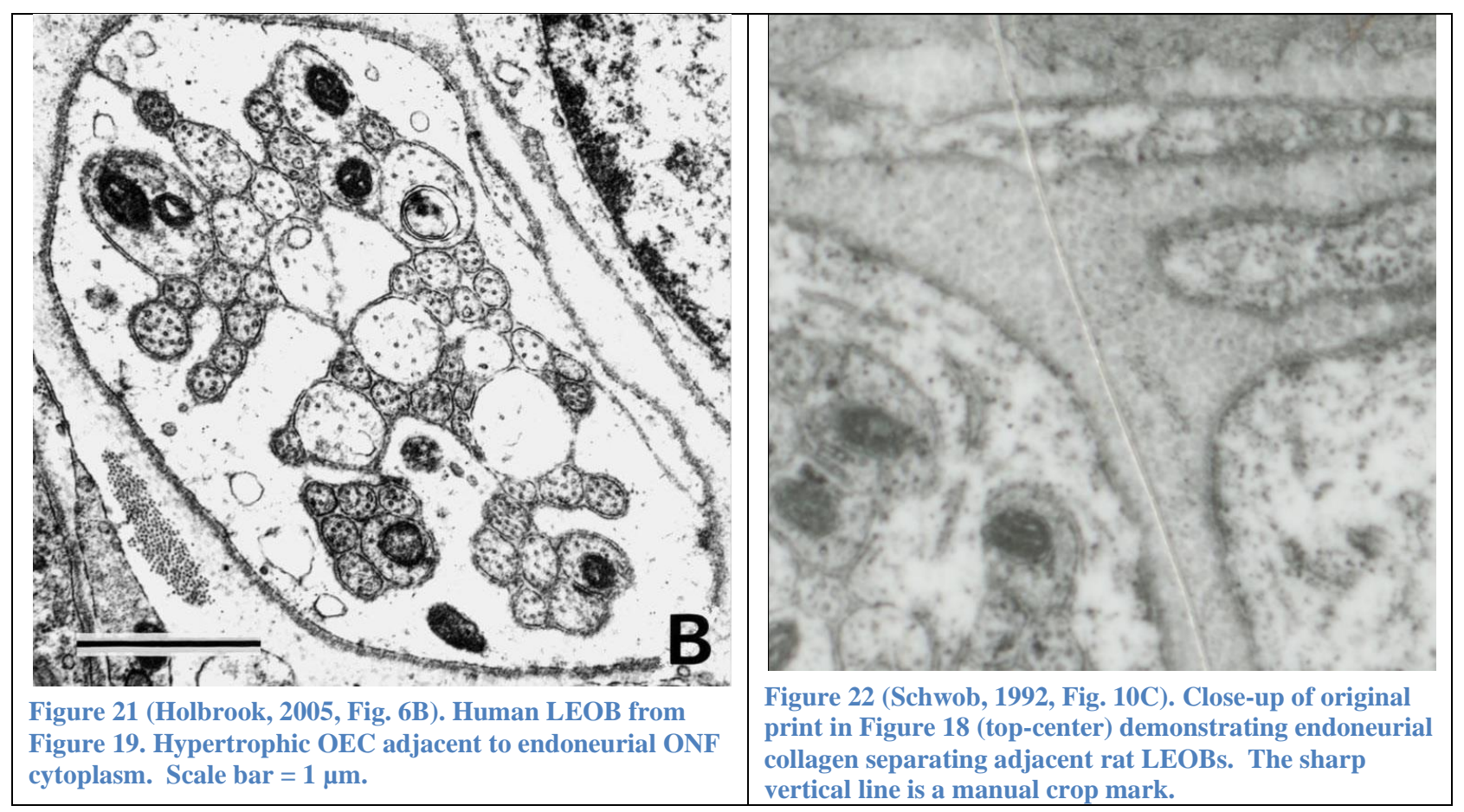




\section{4 - Fascicle Merger}

Kawaja (2009) describes smaller rat fascicles as being composed often of a single LEOB surrounded by collagen and perineurial ONFs, with progressively larger fascicles incorporating an increased number of distinct LEOBs within a single perineurial sheath. This is perhaps most pronounced in the initial portions of the pathway; for example, imagine a fascicle containing a single LEOB exiting the epithelium joining with others like it to form the comparatively larger fascicle depicted in Figure 15. This fascicle contains a number of small LEOBs separated by endoneurial collagen. By necessity, this would be the result of adjacent fascicles joining together, resulting in an increase in the number of LEOBs within that fascicle.

On the other hand, we have described how deeper in the lamina propria these LEOBs also increase in size (Figure 17). By necessity, this would be the result of adjacent smaller LEOBs within a fascicle joining together. This would result in a decrease in the number of discrete LEOBs in that fascicle.

Both of these processes of merger - the joining of separate fascicles, and the joining of their contained LEOBs - are continuous throughout the depth of the lamina propria. The increased size of LEOBs with depth as the result of merger, if it were unaccompanied by the merger of separate fascicles, would make the fascicle observed in Figure 17 impossible.

The relative number of distinct LEOBs within a fascicle does not continuously increase as Kawaja (2009) notes above, but is dynamic: increasing as separate fascicles merge, and decreasing as the contained LEOB merge. The size of the fascicle is correlated to the size of the component LEOBs - thus the absolute quantity of axons - but the number of distinct LEOBs will require an additional deposition of endoneurial collagen and/or ONF cytoplasm between them. 


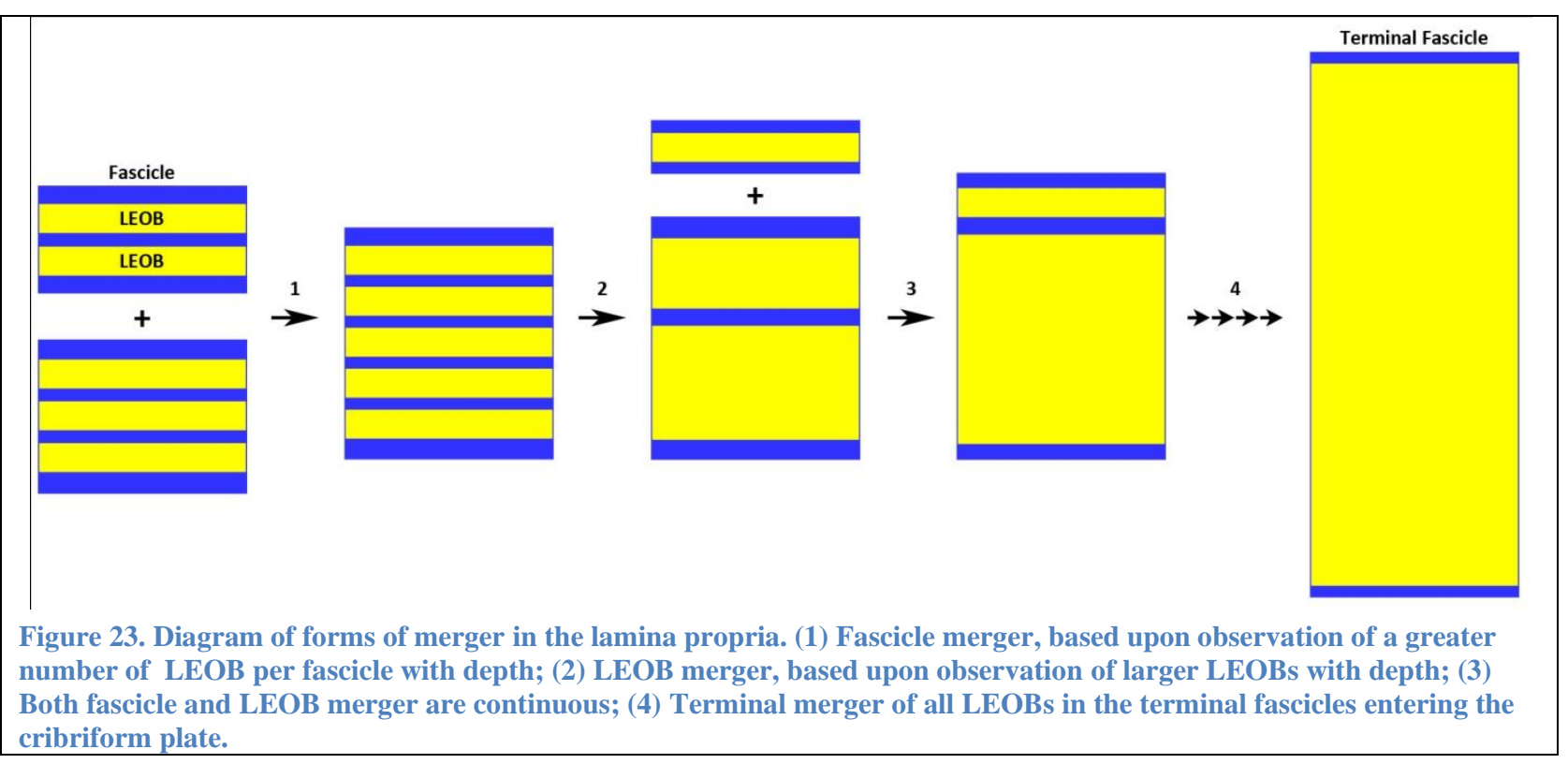

Both types of merger reach a maximum at the level of the cribriform plate, where individual fascicles, containing a single large LEOB, fill each of its foramina (Doucette, 1991). Each terminal fascicle at the cribriform plate can encompass the combined axonal contribution of hundreds of separate LEOBs from the immediate subepithelial region ${ }^{2}$.

The merging of fascicles and LEOBs that culminates at the cribriform plate seems to indicate a gradual process dictated by growth and spatial proximity rather than one that is governed and tightly regulated. It has been shown across 16 species that the area of cribriform plate perforated by olfactory nerves is directly proportional to the area of the olfactory epithelium (Pihlstrom, 2005). Furthermore, a study of bats revealed that - all else being equal larger bats generally had fewer distinct foramina (Bhatnagar, 1974). Thus, a greater area of olfactory epithelium results in a greater area of the cribriform plate being perforated, and a greater distance between the epithelium and the plate allows the fascicles to merge more, resulting in fewer but larger foramina.

All of the morphological permutations observed in the olfactory fascicles are thus likely the result of the sequence in which the pioneering axons converge towards the telencephalon, 
OECs establish ensheathment, and ONFs establish neuria ${ }^{3}$; all three overlap and are subject to the complicated topography of the epithelium from which the axons arise. Our hypothesis might be tested by a detailed quantitative analysis of the fiber pattern across the epithelial sheet towards the bulb.

Of the animals considered, the macaque cribriform plate was reported to be unusual due to the presence of a single large foramen as opposed to multiple punctuate openings (Herrera, 2005). It may have some bearing on the types of mergers achieved in the macaque olfactory nerve: after a certain depth in the lamina propria, these fascicles are described as increasing in size only by the incorporation of a greater number of distinct LEOBs whose size is not described to increase further than the " 500 or more" axons observed more superficially in the lamina propria. This observation was made in passing, so their analysis may neglect the size of the absolute deepest fascicles, but it does raise the question of how progressive merger of fascicles or ONF layering relates to the development of other structures such as the cribriform plate.

Nonetheless, the processes of LEOB and fascicle merger will reach an absolute maximum at the ONL, with all fascicles and LEOBs fusing into this single structure. 
3.5 - Are there interspecies differences in ensheathment style?

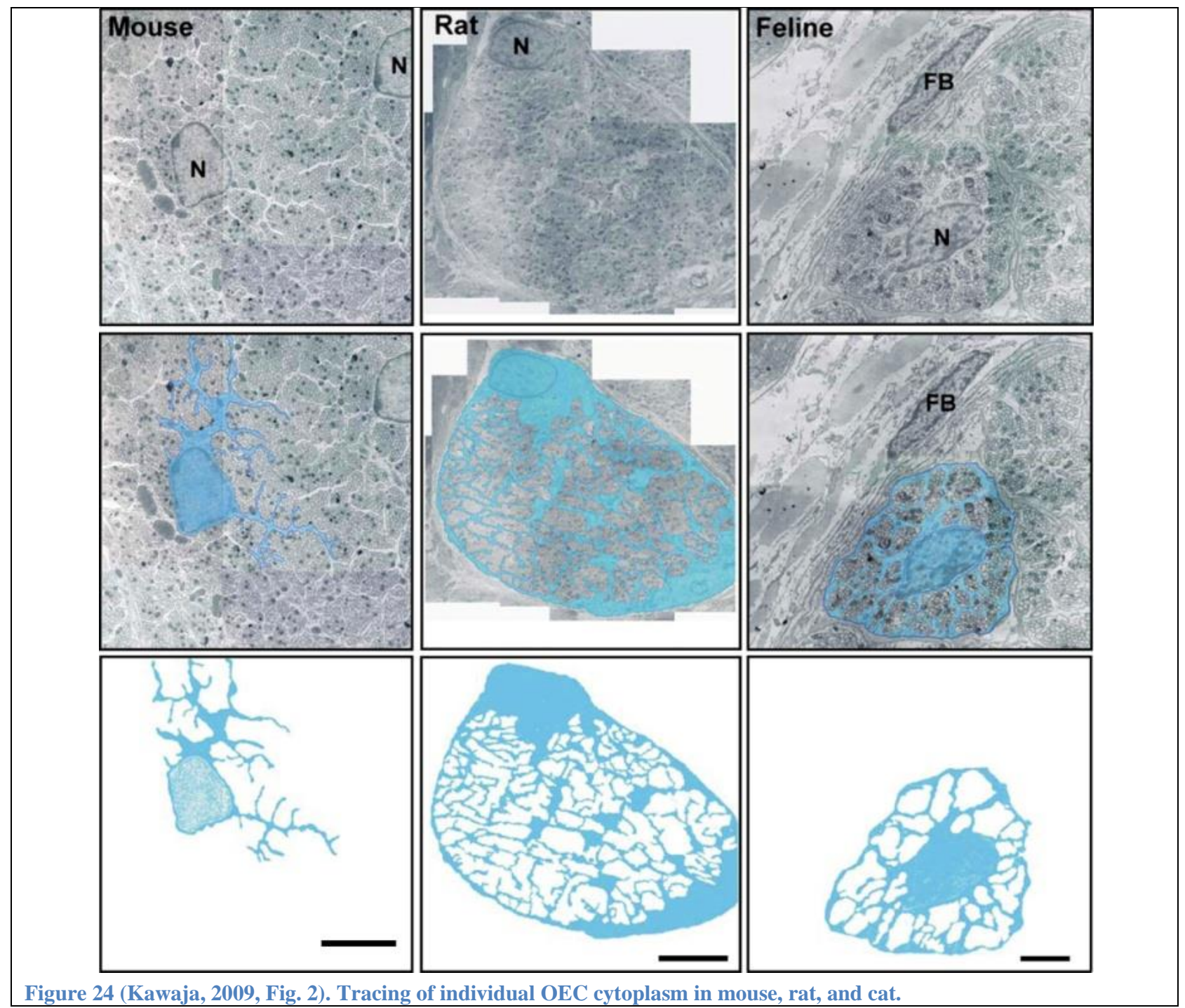

It has been suggested by Kawaja (2009) that there may be interspecies differences among

OECs in relation to the location of their nuclei, and degree of cooperative ensheathment within EOBs in cross-section (Figure 24). It is implied that these may reflect other phenotypic differences relevant to the isolation and experimental manipulation of OECs.

In the literature, OEC nuclei are generally described as being situated at the periphery of EOBs, though they are also noted to be seen elsewhere (Boyd, 2003; Doucette, 1993). Similarly, the cytoplasm of a single OEC can be observed to completely surround a bundle of axons to 
form an EOB, or to coordinate processes with neighboring OECs to do so. Thus, the overall impression is that permutations of both variables - nuclear location and cooperative ensheathment - exist. This impression has not been systematically confirmed, however, within a given species.

\subsection{1 - Interspecies Cooperative Ensheathment}

Kawaja (2009) observes in mice that each olfactory fascicle is composed of a single LEOB, and such LEOB are formed by multiple cooperating OECs per cross-section. In rats, small fascicles also contain single LEOBs, but each is formed by an OEC that individually encloses all of the axons. Larger fascicles in the rat, cat, and rabbit then grow in size by incorporating a greater number of distinct LEOBs. (Although, as reviewed above, it is demonstrable from other reports that these LEOBs also continue to grow in size.) However, it is suggested that OECs in the rat and cat do not demonstrate cooperative ensheathment, and are shown to act individually to ensheath bundles of axons in cross-section (Figure 24). Their analysis implies that fascicles in the mouse increase in size by merging themselves and the contained LEOBs, whereas in the rat and cat the fascicles merge but the contained LEOBs remain distinct.

These purported differences across species do not hold up when other evidence is considered. There are numerous examples of LEOBs in rat that are composed of multiple OECs per cross-section (Field, 2003; Boyd, 2003; Fraher, 1982; Barber \& Lindsay, 1982). There are, of course, also many examples that show an individual OEC per cross-section - many from the same publications (Field, 2003; Fraher, 1982; Li, 2005). In fact, the trend we have described is one of a progressive increase in the number of OECs concomitant with the size of the LEOB. There may also be multiple LEOBs per fascicle (adjacent LEOBs in Figure 17). Since OECs in 
rats engage in cooperative ensheathment as well as ensheathing smaller bundles individually, it is probable that OECs in mice behave similarly and are individually capable of full bundle ensheathment in superficial aspects along the course of the nerve. Thus, the interspecies distinctions regarding cooperative ensheathment made by Kawaja are very likely the result of selection bias. Serial sectioning or other means of defining location as a function of distance from the epithelial origin (i.e. depth within the lamina propria) would be a way to test the relationship definitively.

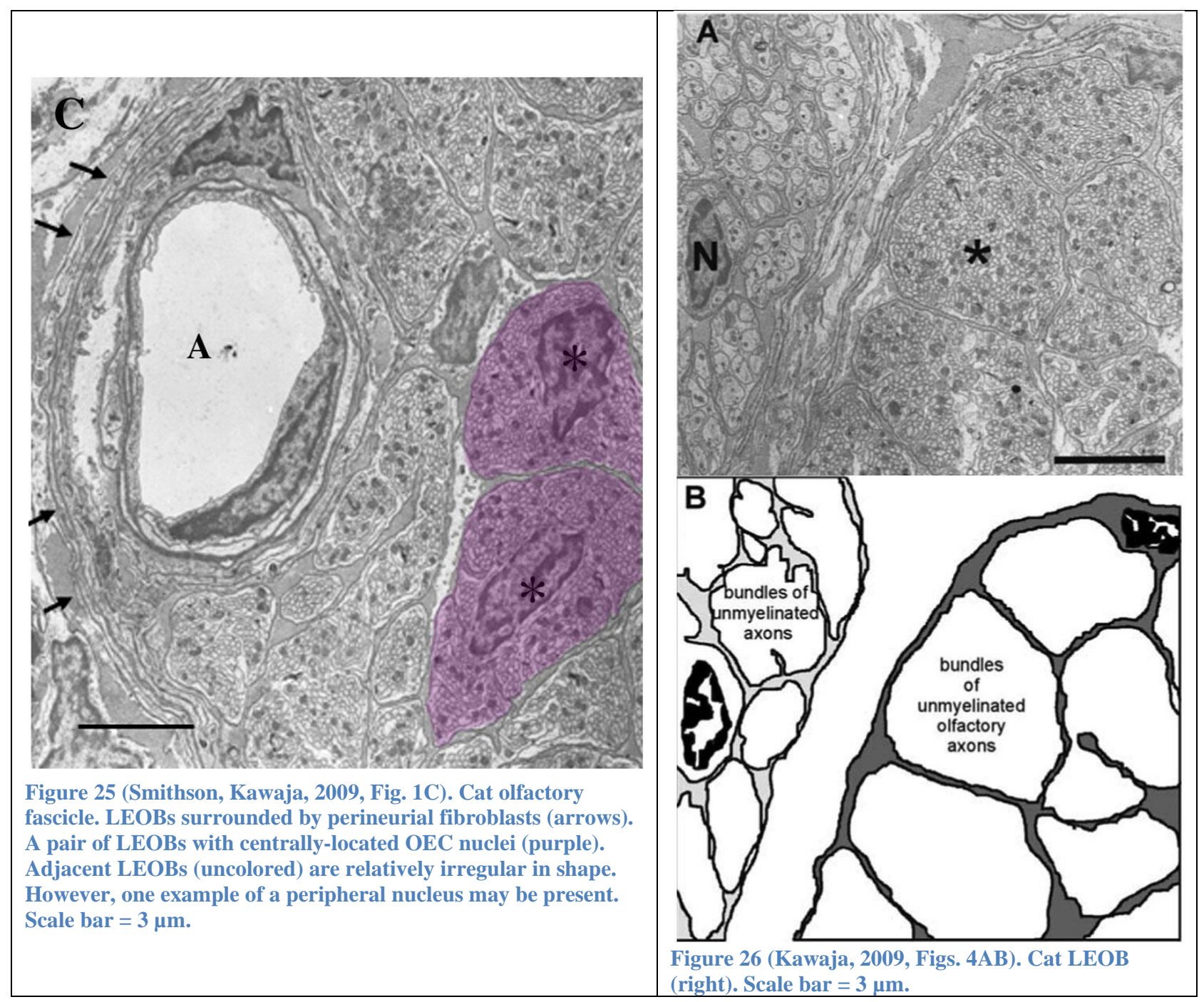

It is also important to note that it is probably not strictly accurate to say that an OEC can accomplish complete ensheathment of a bundle because the interface between two 
longitudinally-adjacent OECs is not a flat plane, but likely involves some cytoplasmic investment from the neighbor when histologically processed into cross-sections (Figure 14). It is always a matter of degree, and thus not likely to be a significant matter of phenotype; this is in contrast to myelinating Schwann cells, for instance, that have well-demarcated nodes separating them.

\subsection{2 - Interspecies Nuclear Location}

Regarding nuclear location, Kawaja (2009) also draws attention to OECs in the cat as having numerous processes radiating away from a centrally-located nucleus (Figure 24). This arrangement is also highlighted in another publication from the same group (Figure 25). This differs from the more typical peripheral nuclear location noted in the literature, although the marginalization of the nucleus is largely based upon EOBs in the rat (Figure 24). Their

illustration of EOBs in the mouse and their description of the manner of ensheathment implies no particular peripheral or central nuclear bias, as it takes many OECs to ensheath the LEOB/fascicles completely. The purpose of distinguishing a central OEC nucleus in the cat is unclear, since other images of cat bundles depict OEC nuclei at a peripheral location (uncolored LEOBs in Figure 25, Figure 26). They do not state the frequency with which the central morphology is observed, nor offer any discussion, other than to imply by presentation that this may be a phenotypic feature of OECs in cats.

A review of the literature reveals that nuclear localization is likely incidental. OEC nuclei in cats may also be seen peripherally (as above). In rats, not only do multiple OECs often comprise each LEOB, but their nuclei are also seen centrally (Figure 15, Figure 27, Figure 28, Figure 29). 


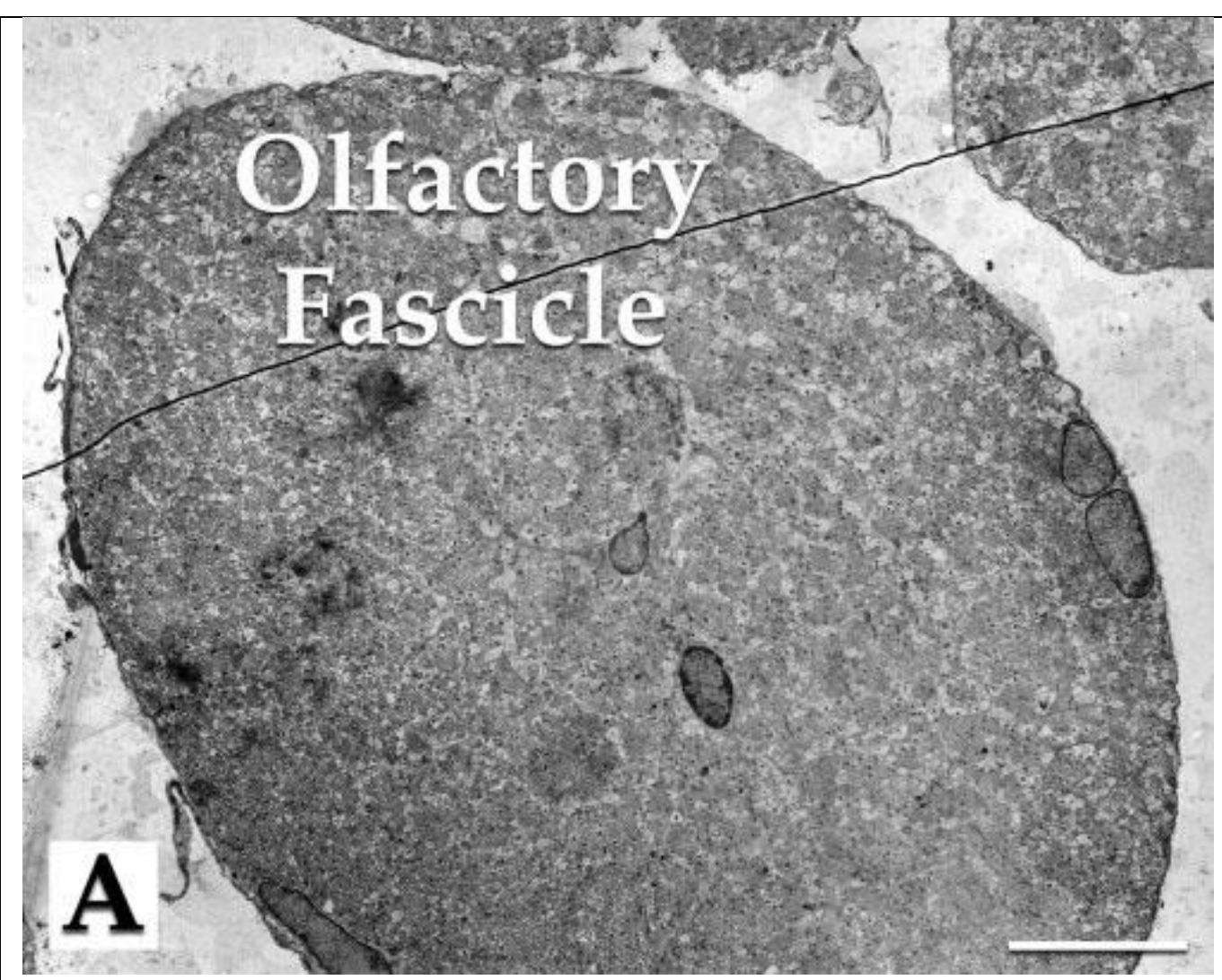

Figure 27 (Boyd, 2003, Fig. 2A). Olfactory fascicle travelling in the subarachnoid space. Note multiple OEC nuclei located both peripherally and centrally. Scale bar $=10 \mu \mathrm{m}$.

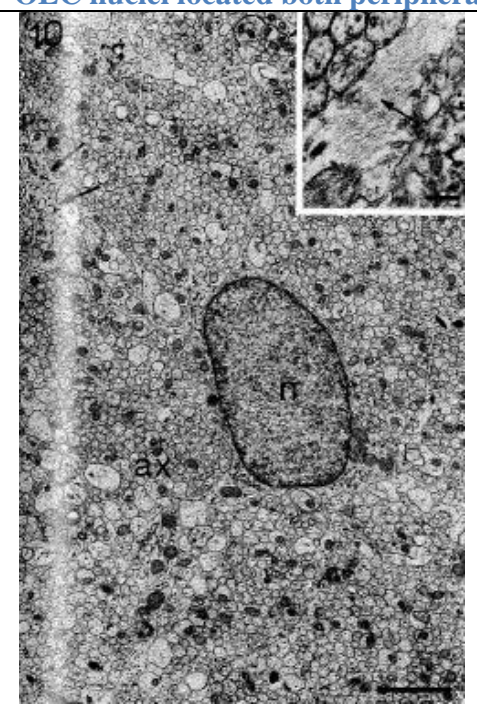

Figure 28 (Barber \& Lindsay, 1982, Fig. 10). Rat OEC nucleus. Scale bar $=2 \mu \mathrm{m}$.

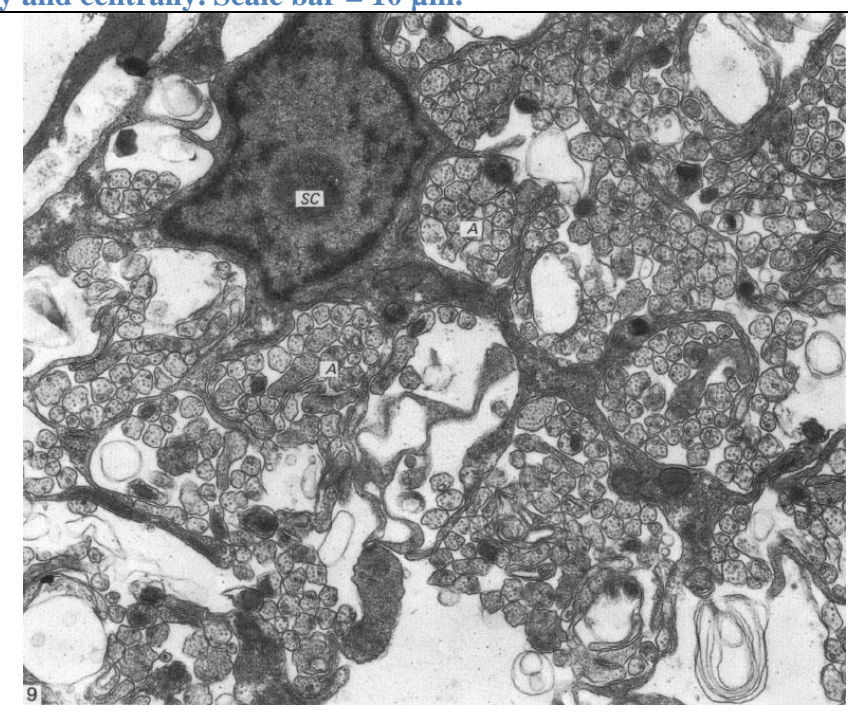

Figure 29 (Doucette, 1983a, Fig. 9). Rat OEC in subepithelial region 27 days after cutting axons on dorsal surface of the bulb. Note $\mathrm{OEC}$ processes and remaining axons surrounding nucleus in all directions. 
Certain examples illustrate how the distinctions between central and peripherally-located nuclei are often unclear (Figure 30, Figure 31, Figure 32). Such ambiguity reduces the likelihood that nuclear location is phenotypically determined, and increases the likelihood that it is the result of dynamic variables such as would occur during growth and development. This point is reiterated in Figure 27, which aptly summarizes the variety of morphologies that may be present in olfactory fascicles and clearly can contain multiple OECs with nuclei in multiple positions. Due to its great depth, it suggests that both these morphological features are primarily dependant upon spatiotemporal variables and not strict considerations of OEC phenotype.

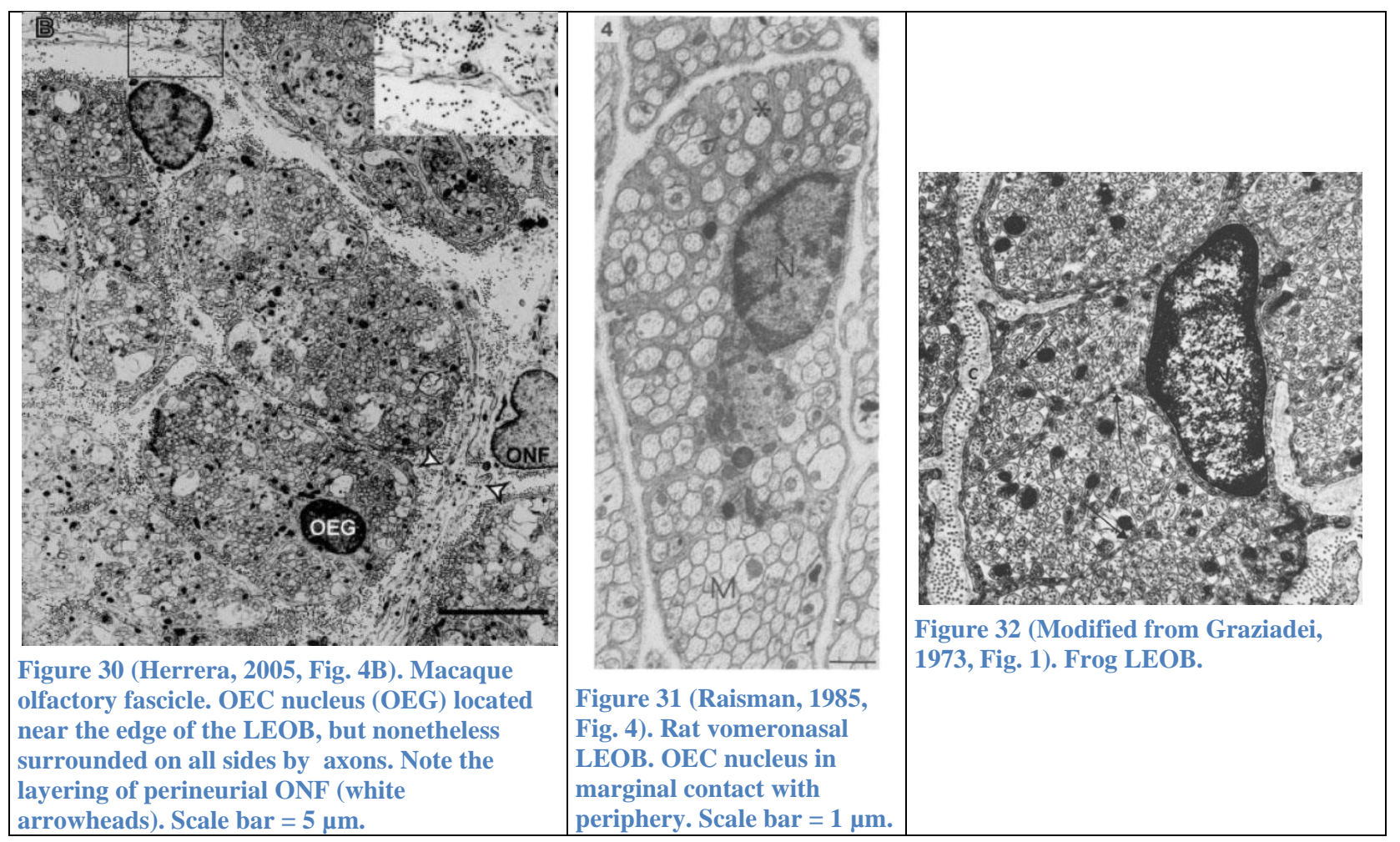




\section{6 - How is OEC nuclear location determined?}

Just as with the emergence of cooperative ensheathment, the manner of axon and pOEC convergence from the placode toward the embryonic telencephalon indicates a possible mechanism for the variety of OEC nuclear localizations observed in EOBs along the length of the projection. In this case, reference will be made to examples as a means of describing possible spatiotemporal mechanisms that give rise to particular observations. These are: (1) peripheral nuclei in small/superficial EOBs, (2) peripheral nuclei in large/distal EOBs, (3) central nuclei in small/superficial EOBs, (4) multi-localized nuclei in very large/distal EOBs.

\section{Peripheral nuclei (Examples 1 \& 2)}

The most frequent observation of peripherally-located nuclei is likely due to the inherent tendency of axons to tightly fasciculate proximally and maintain that association as they extend, combined with the fact that they lead the growth towards the bulb ahead of OECs. Their fasciculation can be observed in vitro (Tisay \& Key, 1999; Hamlin \& Schwob, 2004) and during development (Figure 33, Figure 35).

At the outset, the fasciculation of axons while still in the epithelium (Figure 34) - in its presumably pOEC-free apical region - will necessarily exclude the somata of migratory pOECs from the interior of the smallest bundles entering into the HBC archways (Figure 8) and the immediately adjacent lamina propria. Since the axons lead the migration of pOECs, and the pOECs are not initially tightly associated with them, the degree to which this fasciculation continues in the lamina propria as the axon bundles merge towards the bulb will likewise exclude pOECs from the interior of such bundles. 


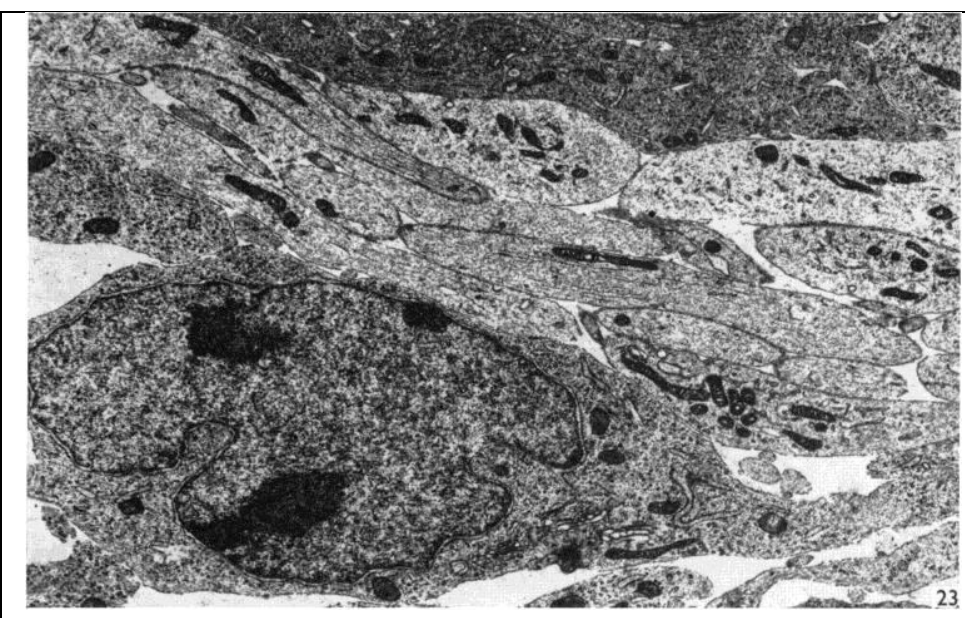

Figure 33 (Cuschieri \& Bannister, 1975b, Fig. 23). Mouse E10 migratory unit in mesenchyme. Bundle of axons in longitudinal section, loosely bordered by $\mathrm{p} / \mathrm{OEC}$.

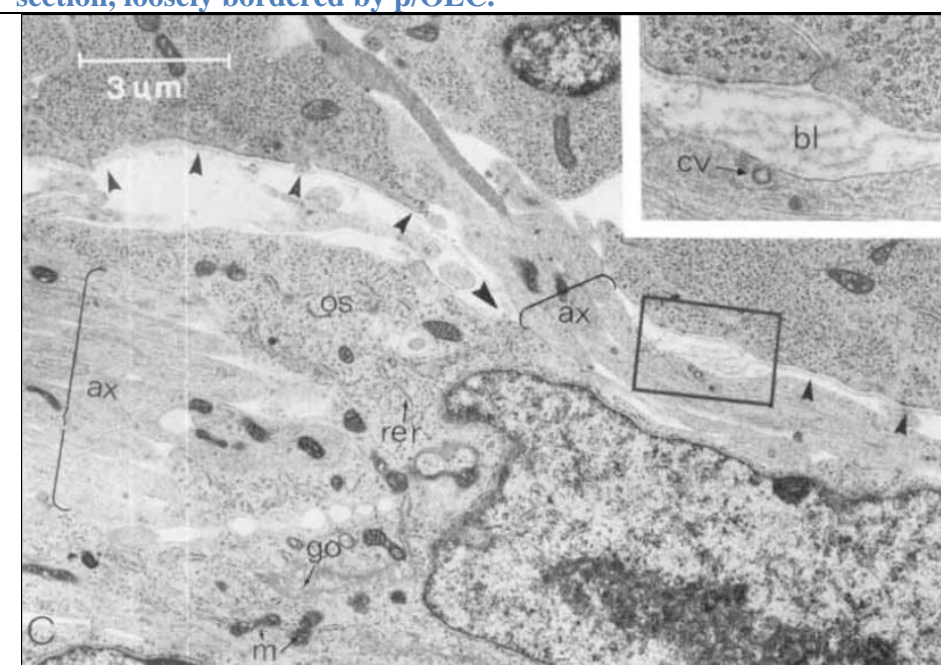

Figure 35 (Marin-Padilla \& Amieva, 1989, Fig. 2C). "Ultrastructural detail of the exit of a few olfactory nerve axons (small ax) through a small opening in the basal lamina (large arrowhead) of the nasal epithelium. Basal lamina anomalies such as bends, blebs, and rolls (inset) are often found at these openings. The intact epithelial basal lamina is outlined by small arrowheads. Also illustrated is a submucosal olfactory nerve fascicle (large ax) surrounded by olfactory sheath cells (os). Coated vesicles (inset), mitochondria $(\mathrm{m})$, rough endoplasmic reticulum (rer), Golgi apparatus (go), and the magnification (horizontal bars) of the figures are also illustrated."

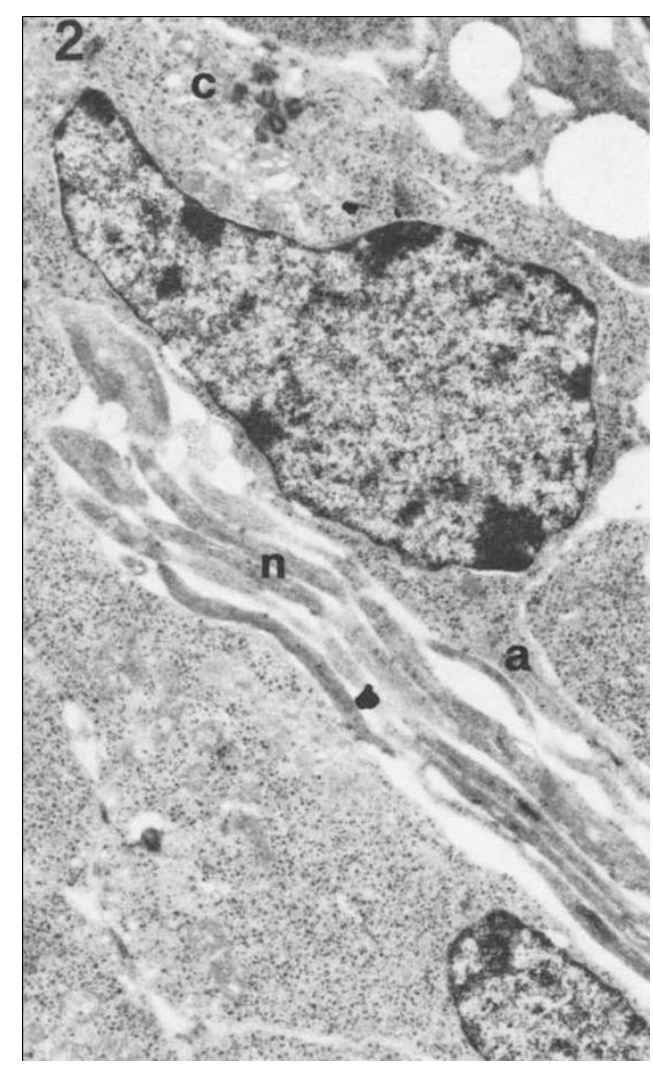

Figure 34 (Farbman \& Squinto, 1985, Fig. 2). Fasciculation of axons during basal descent in epithelium. "Electron micrograph of a developing olfactory receptor cell from an E14 embryo. An axonal process (a) at the basal pole of the cell joins other axons (n) traveling toward the basal lamina. Near the distal pole of the perikaryon several centrioles (c) are seen. $x$ $8500^{\prime \prime}$ 
Imagine a hypothetical scenario in which all of the axons from the epithelium have completed development before any OECs ensheath them. So, all of the axons have extended towards the bulb - having merged and fasciculated in a spatially-defined manner along the way before any OECs are introduced. In this scenario, all of the EOBs would exhibit peripheral nuclei. In reality, the reason that it is only most EOBs that have peripheral nuclei is that this is just partially the case; the OECs only slightly trail the axons, and gradually increase their association along the way. So the predominance of peripheral nuclei is the result of spatial factors - the exemplar of which is the hypothetical "OEC-free" extension of axons. Then, the deviations from this may be the result of temporal factors that are described below.

\section{Central nuclei (Example 3)}

The emergence of centrally-located OEC nuclei in more superficially located portions of the projection may relate to the continued development of the early olfactory epithelium after the initial axons have exited. The pOECs are thought to emerge "in response to the presence of axons in the underlying submucosa" (Marin-Padilla \& Amieva, 1989). Meanwhile, the epithelium continues to expand and new axon bundles continue to exit from it. The pOECs will migrate with the bundles following which they emerge, but will nonetheless also be present in the mesenchyme ahead of subsequent axons exiting from new points in the epithelium. Since in these early stages pOEC will send processes to ensheath any axons in their vicinity (Fraher, 1982; Cuschieri \& Bannister, 1975b), such radial dispositions could be produced when the OEC ensheath axons on all sides.

This is a temporally-based explanation that results from the inversion of the usual sequence - i.e., with pOECs preceding the axons. The central disposition then arises by chance, perhaps aided by the greater surface area from which the axons emerge around the waiting 
pOECs that precludes the possibility of their total fasciculation. Such a mechanism better explains the central nuclei in more superficial EOBs (Figure 15, cat in Figure 24, purple EOBs in Figure 25), as it arises when the later-stage axons enter into the superficial region containing pOECs.

That larger - thus deeper - EOBs tend to exhibit peripherally located nuclei returns to the extent to which axons nonetheless lead the growth and mitosis of pOECs. So, the same EOB with a central nucleus in the subepithelial region may have a peripheral nucleus more distally (Figure 26) because the leading axons will fasciculate ahead of that pOEC's mitosis.

Presumably, no bundles of axons leave the region containing pOECs without acquiring their own contingent, thus the normal order of axons leading the migration of pOECs is restored distally and the peripheral nucleus is again made predominant.

\section{Multi-localized nuclei (Example 4)}

These potential mechanisms must all square with the continuing merger of migratory units leading to the increasing degree of cooperative ensheathment as the bulb is approached. For example, if two large migratory units with peripherally-located pOECs merge, some of these pOECs may be situated between the two and continue to divide along the length of the resultant bundle - their progeny essentially becoming entrapped centrally. They may be admitted within such large bundles because of their sheer size, and/or because as time passes (and thus in more distal regions) the migratory pOECs become more tightly associated with the axons and so are less likely to abandon surface contact with the axons as the two continue to extend and merge with other bundles. 


\section{7 - Primacy of Merger}

Ultimately, the close examination of the data suggests that the myriad morphological distinctions are subordinate to the process of LEOB and fascicle merger. At the level of the cribriform plate, the fascicles reflect cooperative ensheathment with OEC nuclei possible anywhere. But the cribriform plate is not a unique environment, only a landmark. Since evidence exists that multiple species exhibit cooperative ensheathment and multi-localized nuclei at more superficial depths, and all ${ }^{4}$ species eventually do so at the cribriform plate, then these variables probably operate on a spectrum at all depths in all species.

While these explanations of the unfolding of various morphologies are hypothetical, they fit the available structural and developmental observations, and perhaps represent a few simplecase examples of the dynamics at play. The main issue is that any major morphological distinctions across species are not significant, considering a broad accounting of the published observations. The overall picture is that there are a wide variety of morphologies in the olfactory nerves, and these are likely the result of developmental spatiotemporal relationships. The above is an attempt to give an account of what some of the local mechanisms may be. The implication is that the structural development of the projection is not highly programmatic or subject to much complex regulation, but is instead mediated by a few simple principles arising from the source tissue, the olfactory epithelium. Such an autonomous program seems appropriate to the evolutionary age and importance of olfaction, as well as its continued regenerative capacity. 


\section{4 - Subarachnoid Region}

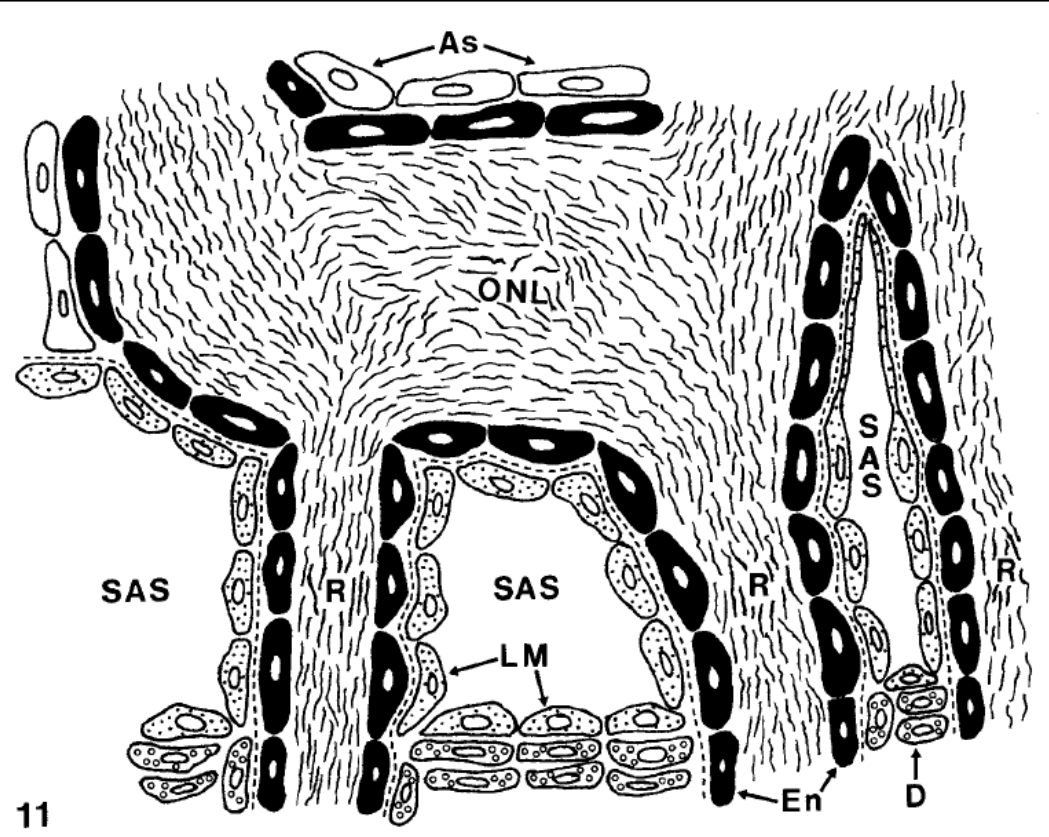

Figure 36 (Doucette, 1991, Fig. 11). Electron-lucent leptomeningeal cells (LMC) surrounding LEOBs to form olfactory rootlets in the subarachnoid space (SAS). Note that in this illustration, the labeling of OEC ("En") is limited, and excludes the OEC nuclei and processes present within the regions labeled "R" and "ONL". Dural cells ("D").

Upon traversing the cribriform plate, the ONFs stop associating with the terminal fascicles and blend into the layer of dural and leptomeningeal cells (LMC) that line the intracranial surface. The ONFs are replaced by LMCs, and these olfactory rootlets fan out in the subarachnoid margin around the rostral bulb. Most of the rootlets fuse immediately on the ventral surface, while others travel greater distances to fuse on its medial, lateral, and dorsal surfaces (Doucette, 1991, 1993a). At the points of rootlet fusion, the LMCs reflect off to become continuous with the pia mater, and the basal lamina of each LEOB reflects off to become continuous with the glia limitans (Julliard, 1998; Doucette, 1991). The EOBs continue into the bulb to form the primary constituents of the ONL.

Most descriptions do not make note of the transition from ONF to LMC ensheathment of the intracranial LEOB as Doucette does - based, at the very least, upon the relative electronlucency of LMCs (1991). It may be important to distinguish between the two cell types because 
they may have different origins. Neural crest (which comes from the neuroectoderm) gives rise to LMCs, while dura and fibroblasts may come from somitomeric-derived mesoderm (although this distinction remains unsettled) (reviewed by O'Rahilly, 1986). Unlike mesodermal fibroblasts, LMCs are reactive to neural tube derived glial factors (Colombo, 1994) and may exhibit distinct interactions with OECs, astrocytes, and the developing bulb. It is most likely that ONFs are of mesodermal origin, but this is not known for certain. However, it has been noted that the LMCs do not express external basal lamina, while perineurial ONFs express it on various surfaces (Herrera, 2005).

It has been suggested that ONFs play a role aiding OECs in the maintenance of open channels in the lamina propria through which axon regeneration takes place (Li, 2005; Raisman, 2001). A number of OEC/ONF coculture and transplant experiments have been undertaken to examine this possibility. Unfortunately, by neglecting the transition from ONFs to LMCs in the subarachnoid space, most of these protocols utilized LMCs instead of ONFs (Li, 1997, 1998, 2003; Deumens, 2004, 2006). The fibroblastic cells on the surface of the bulb, the LMCs, were assumed to be the same as the fibroblastic cells in the lamina propria, the ONFs. Since they most likely are not, the LMCs were studied under the false pretense of OEC-aiding properties originally attributed to ONFs in the lamina propria. While this does not invalidate particular experimental results, it does affect their subsequent interpretation since proper cellular identities were not recognized. This recognition may resolve the observation in these studies of the presumed ONFs appearing to share properties of astrocytes as well as fibroblasts (Li, 1998; Deumens, 2004). In fact, LMCs have the electron-lucent appearance of astrocytes, but express fibronectin when exposed to astrocyte-conditioned media (Colombo, 1994) as occurs in these CNS transplant models. To examine the original aims, these coculture experiments bear 
repetition with ONF proper. On the other hand, it may turn out to be serendipitous and LMCs are more useful than ONFs for co-transplant with OECs into areas of CNS injury. This would not be entirely surprising, as the neuroectodermal origin of LMCs may make them more inherently compatible with such an environment.

Another reason to heed the transition from ONFs to LMCs is that it may represent a concrete means by which to define the transition from the PNS to the CNS compartment, which is otherwise complicated by the fact that the OECs seamlessly transect the two. Elsewhere in the nervous system, the cast of glial cells is sharply delineated - with Schwann and satellite cells peripherally, and oligodendrocytes and astrocytes centrally. Investigation of interactions of OECs with ONFs, dural cells, and LMCs may reveal factors that lead to a more fundamental description of these two compartments. This could shed light on why the other glial types are so clearly divided, how the OECs manage to be intermediate, or more esoteric possibilities such as OECs - and the other cells from the olfactory placode - being inherently CNS tissues. The latter possibility being raised because the bulb and the epithelium are totally physically continuous, and by an intriguing study of zebrafish development that demonstrated that the cells of the olfactory placode migrate from the neural plate, rather than differentiating in situ from an isolated set of neural crest cells (Whitlock, 2004).

Doucette $(1991,1993 a)$ noted in the rat that some of the rootlets penetrate deeply into the ONL before losing their LMCs and basal lamina. This may have implication for experimental procedures that rely on manual dissociation (or "peeling off") of the leptomeninges to expose the ONL for harvesting of OECs. Such manipulation may tear these deeply penetrating rootlets along the continuous arc of the ONL border, and thus retain contaminating streaks of LMCs within the ONL. These fissures were deepest on the ventral surface of the ONL, and imparted a 
highly convoluted appearance upon it (Doucette, 1991). The dorsal, medial, and lateral surfaces also have a convoluted appearance, but this is because the long-travelling rootlets from the ventrally-located cribriform plate fuse with these surfaces of the ONL at acute angles (Doucette, 1993a), thus resulting in rootlets that are only partially fused when viewed histologically (Julliard, 1998).

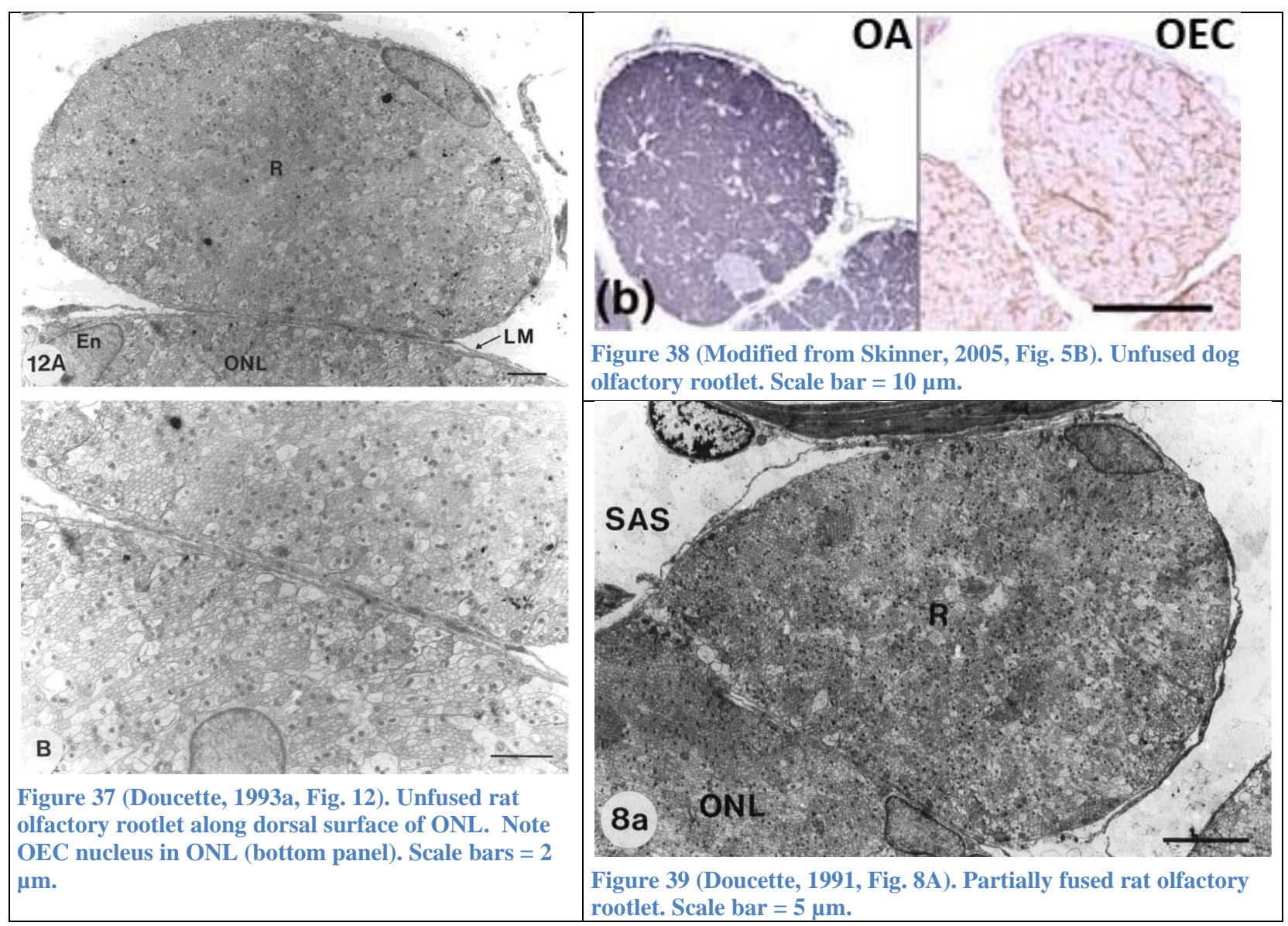




\section{5 - Olfactory Nerve Layer}

The ONL is the outermost layer of the bulb into which every olfactory rootlet fuses. It consists primarily of OECs and olfactory axons, and may be considered a terminally large EOB containing all of the axons from the ipsilateral epithelium, so the axons are no longer isolated from one another by fibroblasts or other connective tissue; they are firmly in the CNS compartment. The OECs continue to ensheath the axons, but do so in a looser manner and no longer express basal lamina around individual bundles. The ONL is thus a large cooperativelyensheathing OEC matrix through which the axons travel. The processes emanating from an individual OEC may ensheath multiple axon bundles (Figure 40). Conversely, an individual bundle may be ensheathed by processes from multiple OECs in a single cross-section (Valverde, 1991; Au, 2002; Rela, 2010). This is similar to the manner of ensheathment in the lamina propria during embryonic development prior to the formation of basal lamina (see Section 3.1). The dynamic morphology of these OECs may be mediated by complex lamellopodial waves (Windus, 2007, 2010) and varying degrees of gap-junction connectivity between neighboring OECs (Rela, 2010).

\section{Glia Limitans}

Elsewhere in the CNS, the glia limitans is formed by astrocytes. In those regions of the ONL penetrated by olfactory rootlets (transitional zones), the glia limitans is formed exclusively by OECs (Figure 36). Astrocytes are only seen contributing to the glia limitans at a distance from these zones (Doucette, 1991). Where the OECs and astrocytes abut one another, there is never any intervening basal lamina (Doucette, 1993a). The dorsal surface of the ONL has the fewest penetrating rootlets, so the majority of the glia limitans here is formed by astrocytes. In contrast, 
the ventral surface is extensively penetrated by olfactory rootlets, so the glia limitans is almost entirely formed by OECs.

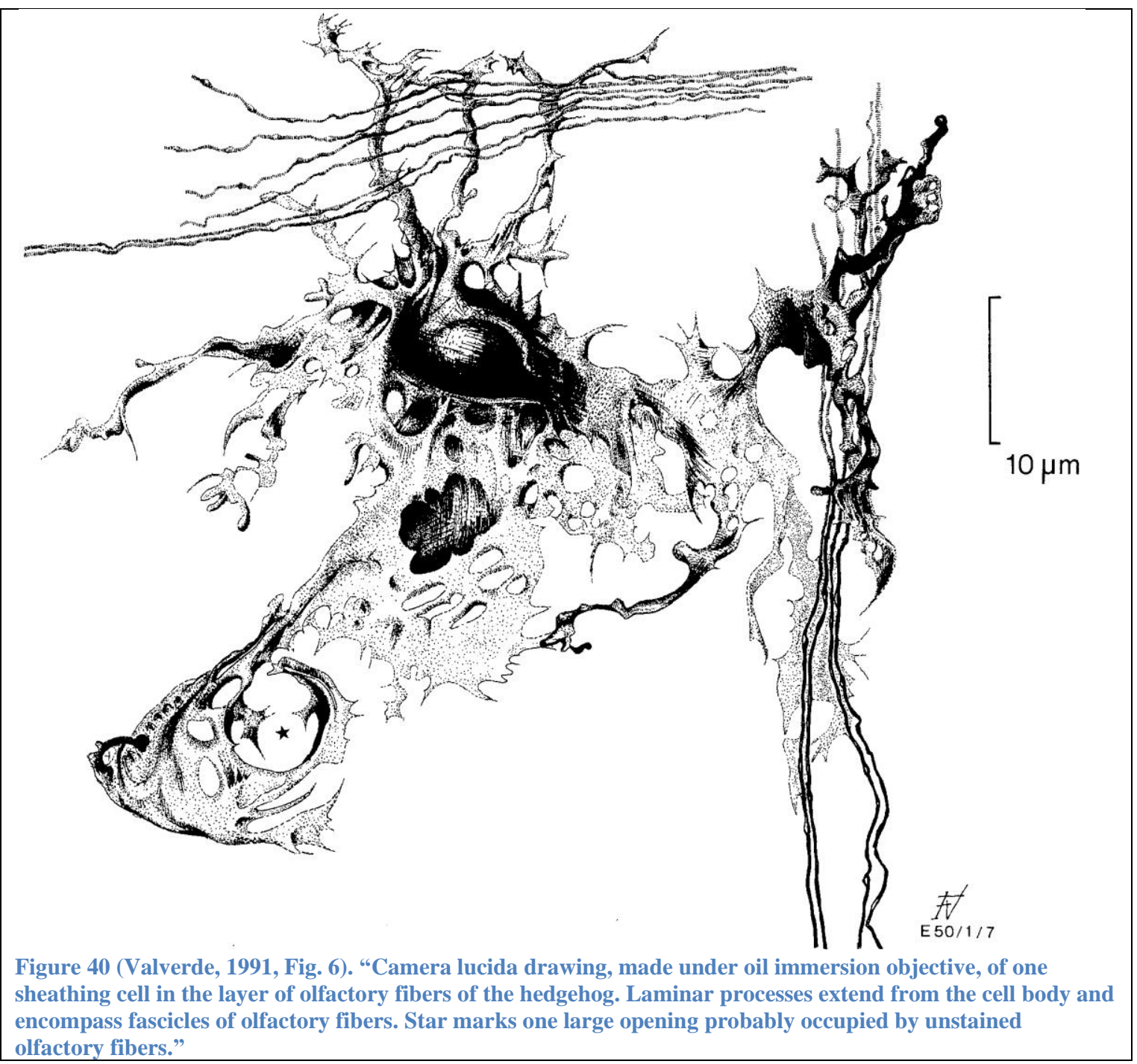

\section{Astrocytes}

The presence of astrocytes between EOBs in the ONL implicate it as a CNS region. The astrocytic processes may sometimes come into contact with the axons, but they never fully ensheath them (Doucette, 1984; Herrera, 2005). So, the OECs do not totally isolate the axons from the other constituents of the ONL, as had been suggested by Raisman (1985). It has been noted that these astrocytes are not present until some time after birth (Doucette, 1993a). They 
appear to migrate from the deeper bulb postnatally, rather than differentiate from progenitor cells in the ONL (Doucette, 1993b).

\section{OEC basal lamina}

In the ONL, OECs only produce basal lamina on those surfaces facing the glia limitans. The expression of basal lamina on the mesenchymal faces of the OECs is therefore identical to the OECs of the lamina propria. This observation may shed some light on the question of how the absence of a basal lamina around the remainder of the OECs in the ONL is mediated. One suggestion was that the absence of basal lamina on these OECs was phenotypic - that these cells were a distinct sub-population (Franceschini, 1996). However, prior to that, Doucette (1990) had wondered whether the astrocytes in the ONL were responsible for directly modulating the production of OEC basal lamina. So, OECs constitutively produce basal lamina, and astrocytes inhibit this. The converse possibility would be that OECs do not produce basal lamina unless induced to do so, perhaps by fibroblasts. Since we have observed that intraepithelial OECs also lack basal lamina (see Section 2), the possibility of fibroblastic induction seems the most likely since there are no astrocytes in the olfactory epithelium. It also makes OEC's production of basal lamina consistent from the epithelium to the ONL, so there would seem to be no reason to posit a distinct sub-population on this basis. That OECs produce basal lamina on those surfaces exposed to fibroblastic cells has the additional virtue of helping to explain how cooperatively-ensheathing LEOBs in the lamina propria mediate the production of basal lamina only on the outer perimeter, and thus specifically how cooperative ensheathment - as defined by a single basal lamina surrounding multiple OECs - itself arises. 


\section{1 - Laminarity: Axon Sorting}

On the approach to the ONL, the axons within EOBs are intermixed with regard to OR type - they are heterotypic bundles (St. John, 2003). Within the ONL, they defasciculate, rearrange, and refasciculate with axons expressing the same OR (Chehrehasa, 2006; Feinstein \& Mombaerts, 2004; Mombaerts, 1996); these homotypic bundles then target specific glomeruli. The ONL has two visible outer and inner laminae, and within it occurs the transition from the large heterotypic bundles of the olfactory rootlets to the multiple homotypic bundles that target specific glomeruli in the underlying glomerular layer.

In the outer ONL (ONLo) the axon bundles appear generally well-defined by OECs and travel parallel to the surface of the bulb, extending around it in a geodesic-like path from their points of fusion predominantly on the ventral surface. It is considered that axon extension and regional targeting occur primarily in this layer (Au, 2002; Treloar, 2002). These bundles appear relatively distinctly (Figure 45) - depending on the overall density of rootlets fusing on that particular surface of the bulb - but they are heterotypic and so contain axons expressing a variety of OR types (Figure 44A).

In the inner ONL (ONLi), the axons defasciculate and refasciculate into the homotypic bundles that synapse on specific glomeruli (Au, 2002; Treloar, 2002). Here the axons exhibit a more complex set of trajectories and are no longer necessarily travelling parallel to the contour of the bulb as they reassort and enter obliquely into the underlying glomerular layer. Likewise, the processes of OECs do not ensheath sets of axons into the more orderly bundles seen in the ONLo (Au, 2002). It may be said that the OECs in the ONLo more specifically ensheath bundles of axons, while in the ONLi they form a looser matrix through which axons travel as necessary. 


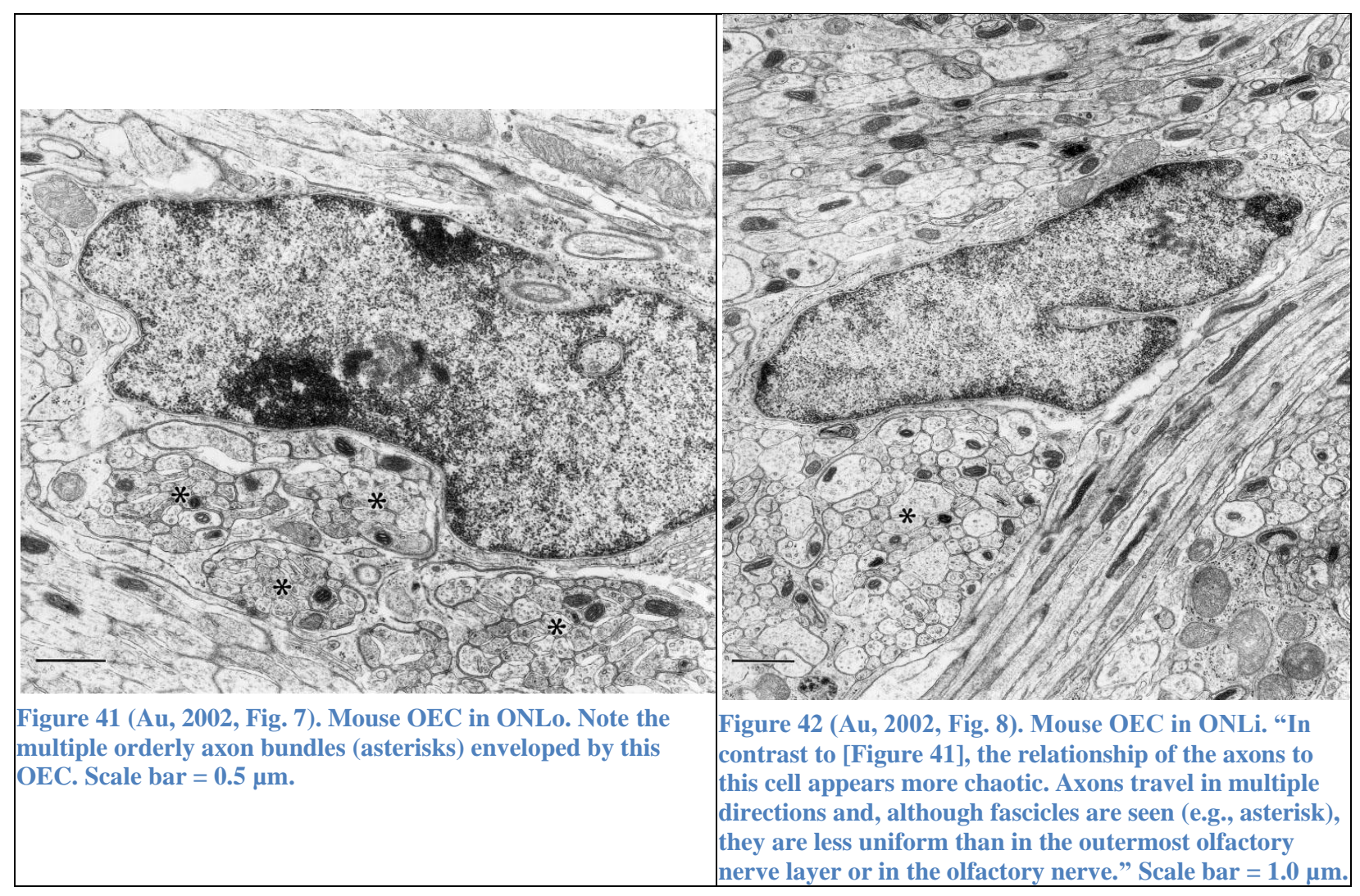

The best way to apprehend the difference between the ONLo and the ONLi is to view the immunochemical markers for a specific subset of axons (Figure 43, Figure 44) in comparison to that of all mature axons (Figure 45, Figure 46). The ONLo is more orderly when looking at all mature axons (Figure 45), but less so if looking at just one subset (ONLo, Figure 43); the bundles are more distinct, but heterotypic. The ONLi is more orderly when looking at just one subset of axons (ONLi, Figure 43), but less so if looking at all mature axons (Figure 46); the bundles are more homotypic, but it is a dense and complex region. 


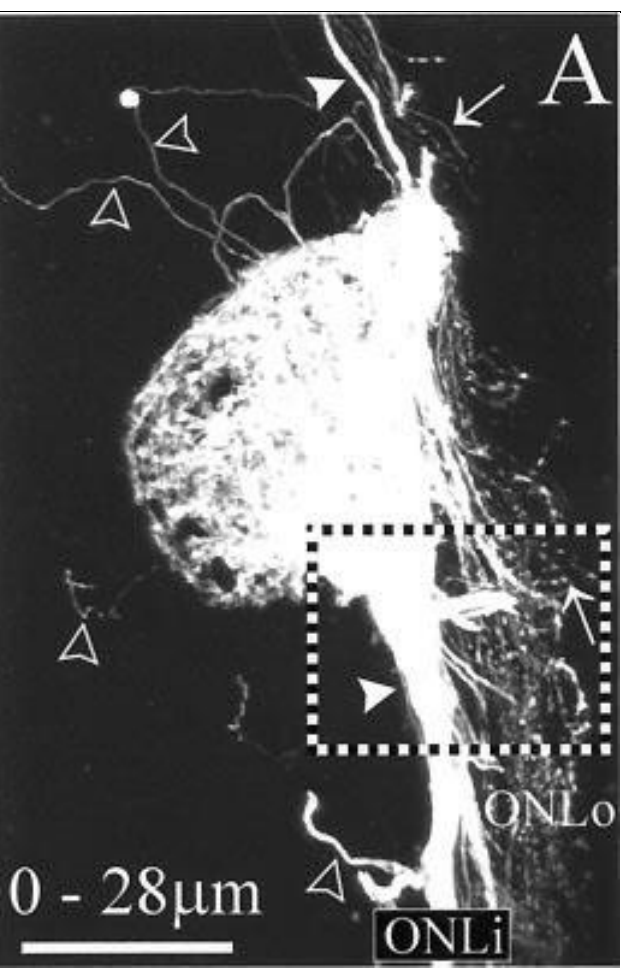

Figure 43 (Treloar, 2002, Fig. 1A). Trajectory of axons expressing a single OR gene (M72) in adult mouse bulb. Notice the diffuse pattern of the M72 axons in the ONLo (right), suggests their travel in different heterotypic bundles. In the ONLi, the M72 axons have coalesced into distinct homotypic bundles targeting the glomerulus.

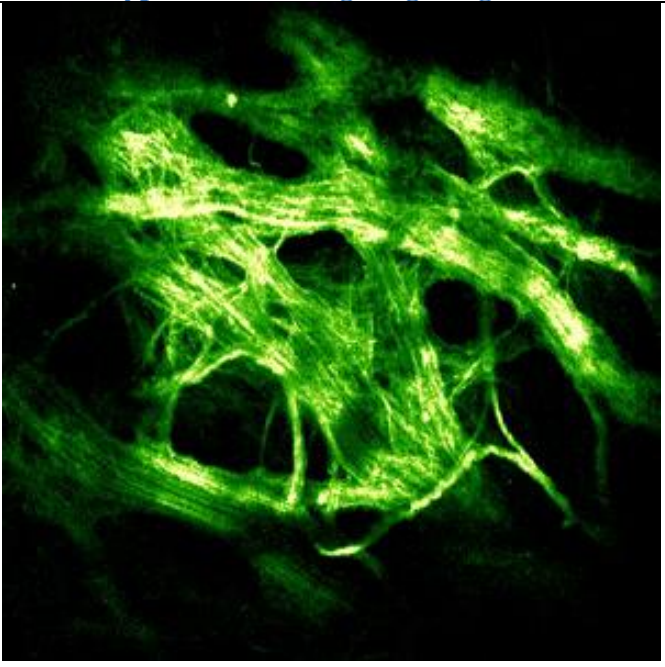

Figure 45 (Potter, 2001, Animation 3, Frame 7).

Trajectory of all mature axons (expressing OMP) in the ONLo. A comparison of the discrete bundling of all axons in this image to the diffuse appearance of M72 axons in Figure 43 (right side) highlights the heterotypic nature of bundles in the ONLo.

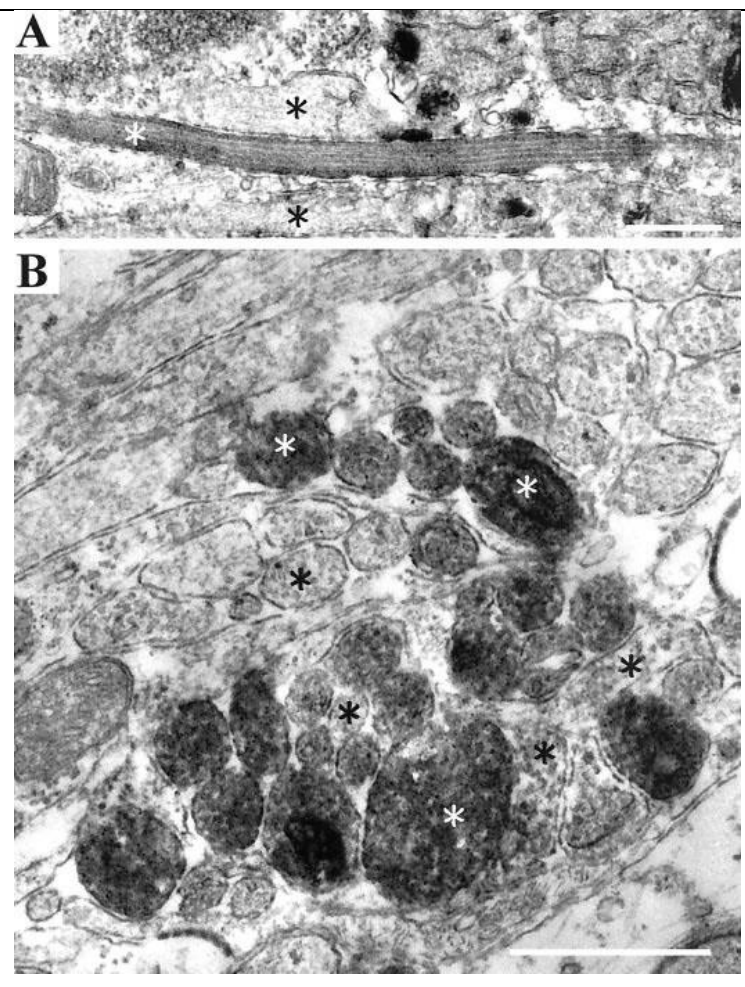

Figure 44 (Treloar, 2002, Figs. 2AB). Ultrastructural immunolocalization of GFP-expressing M72 axons. (A) Single M72 axon (white asterisk) adjacent to non-M72 axons (black asterisks) in ONLo. (B) More homotypic M72 bundle in the ONLi. Scale bar $=0.5 \mu \mathrm{m}$.

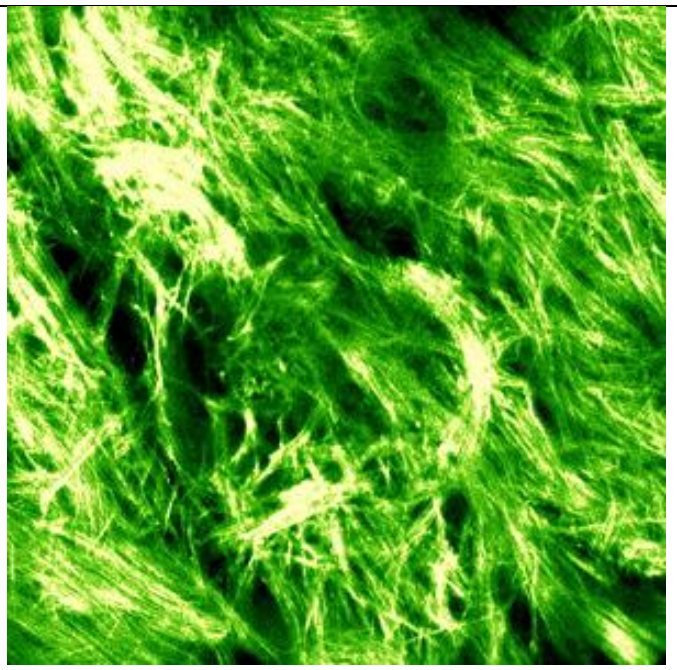

Figure 46 (Potter, 2001, Animation 3, Frame 15).

Trajectory of all mature axons (expressing OMP) in the ONLi. Though there are more axons travelling in multiple trajectories, they have accomplished some degree of sorting into homotypic bundles. 
In addition:

However, the NFL [nerve fiber layer, ONL] is not a uniform structure but varies considerably with anatomical location. The rostral and ventral NFL is relatively thick with distinct inner and outer layers, whereas the NFL in the dorsal and caudal regions is thin and without the distinct layers observed in the rostral and ventral NFL. Most of the defasciculation and sorting of axons occurs when the axons first enter the rostral and ventral NFL [Au, 2002; Treloar, 2002]; axons in the dorsal and caudal NFL are largely already sorted and are projecting to their target glomeruli [St. John \& Key, 2001]. (Windus, 2010)

It was previously described that the majority of olfactory rootlets fused on the ventral

bulb, with some travelling to the medial, lateral, and dorsal surfaces (see Section 4).

Additionally, these areas are mostly confined to rostral portions of the bulb (J.E. Schwob,

personal communication). So it may be said that there is only an ONLo in the regions where the rootlets fuse.

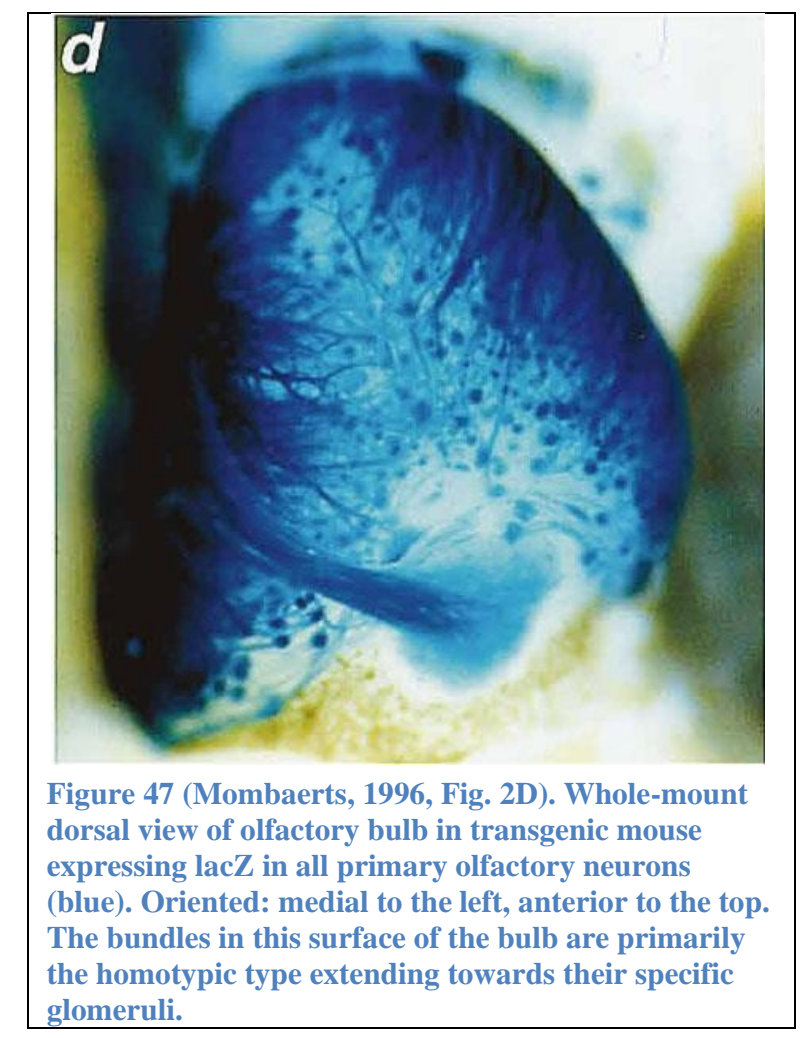


It appears that in the ONLo, the well-fasciculated heterotypic bundles seem primarily to be extending. whereas the ONLi is where the real "work" of reorganization appears to take place: the defasciculation, rearrangement, and glomerular targeting. It should be noted that the first two-thirds of this "work" appears to occur entirely in the rostral ONLi, since the above excerpt describes the caudal ONLi as only completing the last step of glomerular targeting/extension; the same applies to the dorsal ONLi (Figure 47) as the feature is dictated more by the distance from the rostral and ventral extent of the ONL.

The morphology of the ONL, in general, is postulated to arise as the growth cones of axons dynamically sample other axons for similarity or dissimilarity (Feinstein \& Mombaerts, 2004). Such a description is better evinced by the morphology of the ONLi. Similarly, the experiments that demonstrated the ability of the olfactory epithelium to form ectopic glomeruli also describe the formation of an axonal plexus that better resembles the ONLi (Figure 48; Graziadei, 1978, 1979; Graziadei \& Monti-Graziadei, 1986). None demonstrated the outer/inner laminarity observed in the normal rostral ONL. Even when the bulb is unilaterally removed and replaced with a bulb-shaped artificial scaffold, rudimentary ONL and glomeruli form, but there is no laminarity to the ONL itself (Chehrehasa, 2006). The complex morphology of these axonal plexuses, as well as their adjacency to the ectopic glomerular structures, closely resemble the structure and function of the normal ONLi, but not the ONLo. 


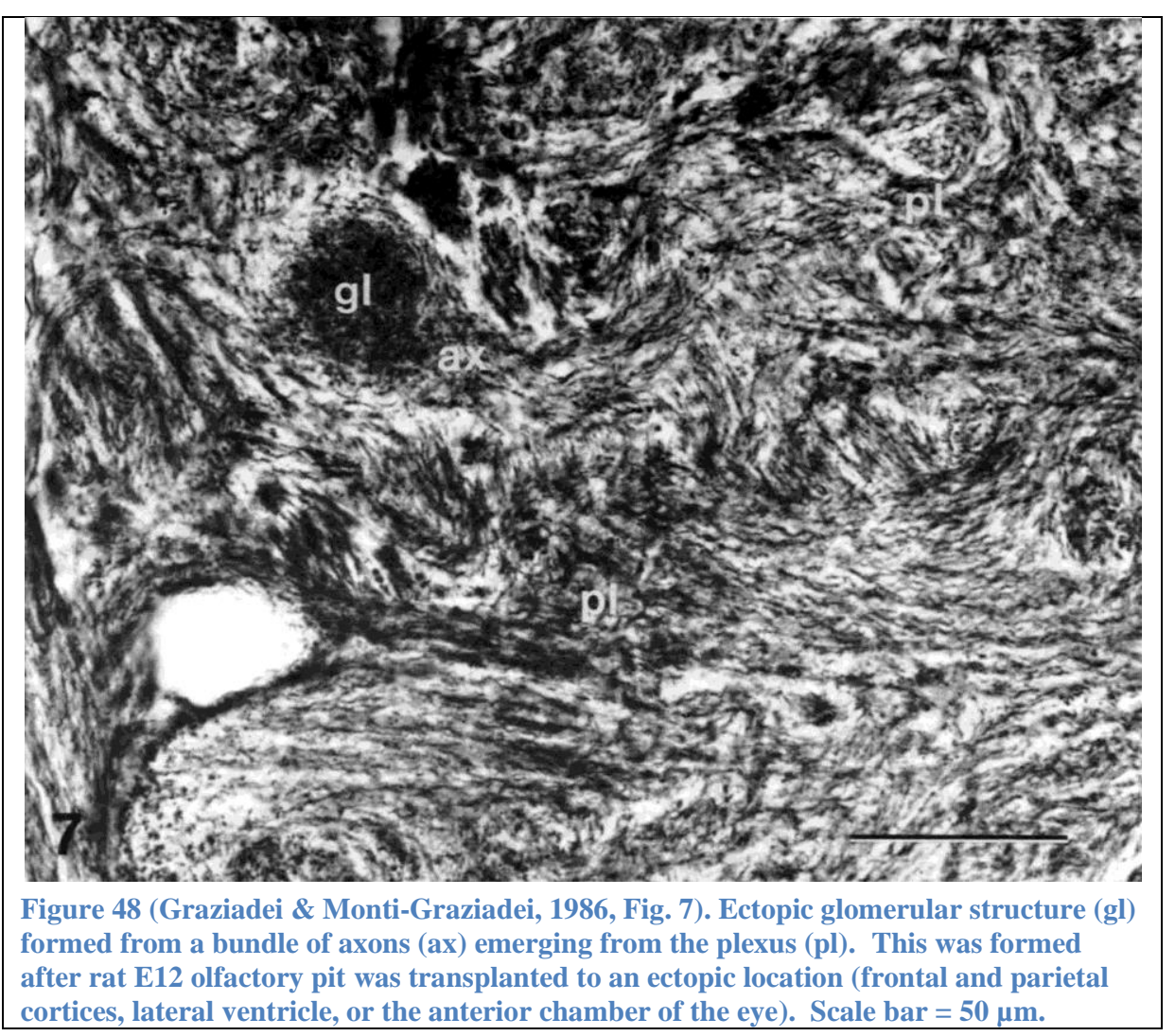

The details of axonal targeting and the formation of functional olfactory glomeruli in the bulb are the subject of ongoing and complex investigation beyond the scope of this review.

Nonetheless, even in this regard, Feinstein \& Mombaerts (2004) propose the view that "axons do not converge onto a glomerulus, but coalesce into a glomerulus". So the functional development of glomeruli in the olfactory bulb is not intrinsically different than what is seen in the formation of glomeruli in ectopic substrates. In addition, the coalescence of axons into glomeruli in both normal and ectopic settings is associated with a plexus of remarkably similar morphology.

These details are, however, relevant here insofar that the ONL is largely composed of OECs, and these are intimately related to the axons. Thus, the laminarity of the ONL as it pertains to axons also pertains to OECs. In addition, there are morphological and biomolecular differences between OECs in the ONLo and ONLi (Au, 2002; Franceschini, 1996; Astic, 1998; Treloar, 1999; Gong, 1994). Though such variations may sometimes compel the definition of 
OEC subtypes or other "OEC-like" cells, we have considered all such cells to be OECs on the basis of their continued ensheathment of the axons. This is best supported by observations in transgenic mice expressing DsRed-human S100 $\beta$ promoter in OECs that demonstrate the same cells comprising the olfactory fascicles and ONLo entering well into the ONLi - even in an area where the OECs no longer express endogenous S100 $\beta$ protein (Windus, 2007, 2010). Since all of the ensheathing cells are lineally related, and their morphological and biomolecular features are subject to some plasticity during development and in vitro (Astic, 1998; Franceschini, 1996; reviewed in Ramon-Cueto \& Avila, 1998; Wewetzer, 2005), it is probably best to embrace this definition of OECs based upon their ensheathment of the axons. Since this relationship is maintained throughout the formation of olfactory projection, the question of what causes the variation of OEC properties across the laminae of the ONL becomes associated with what causes the distinct projection patterns of the axons in these laminae, and both seem bound to the details surrounding the initial formation of the bulb.

\section{2 - Why is there such a distinct ONLo?}

We may begin by asking: What is the cause or purpose of a notably thick region confined to the rostral and ventral extent of the bulb? This seems related to the fact that the rostral and ventral ONL is the primary area of rootlet fusion with the bulb, so this is the region where the majority of the functional processes of the ONL would take place. But then the question becomes of this region: Why is there a outer lamina where the axons remain in distinct heterotypic bundles? Why do the bundles here not more rapidly undergo the functional processes apparent in the ONLi or ectopic axonal plexuses? 


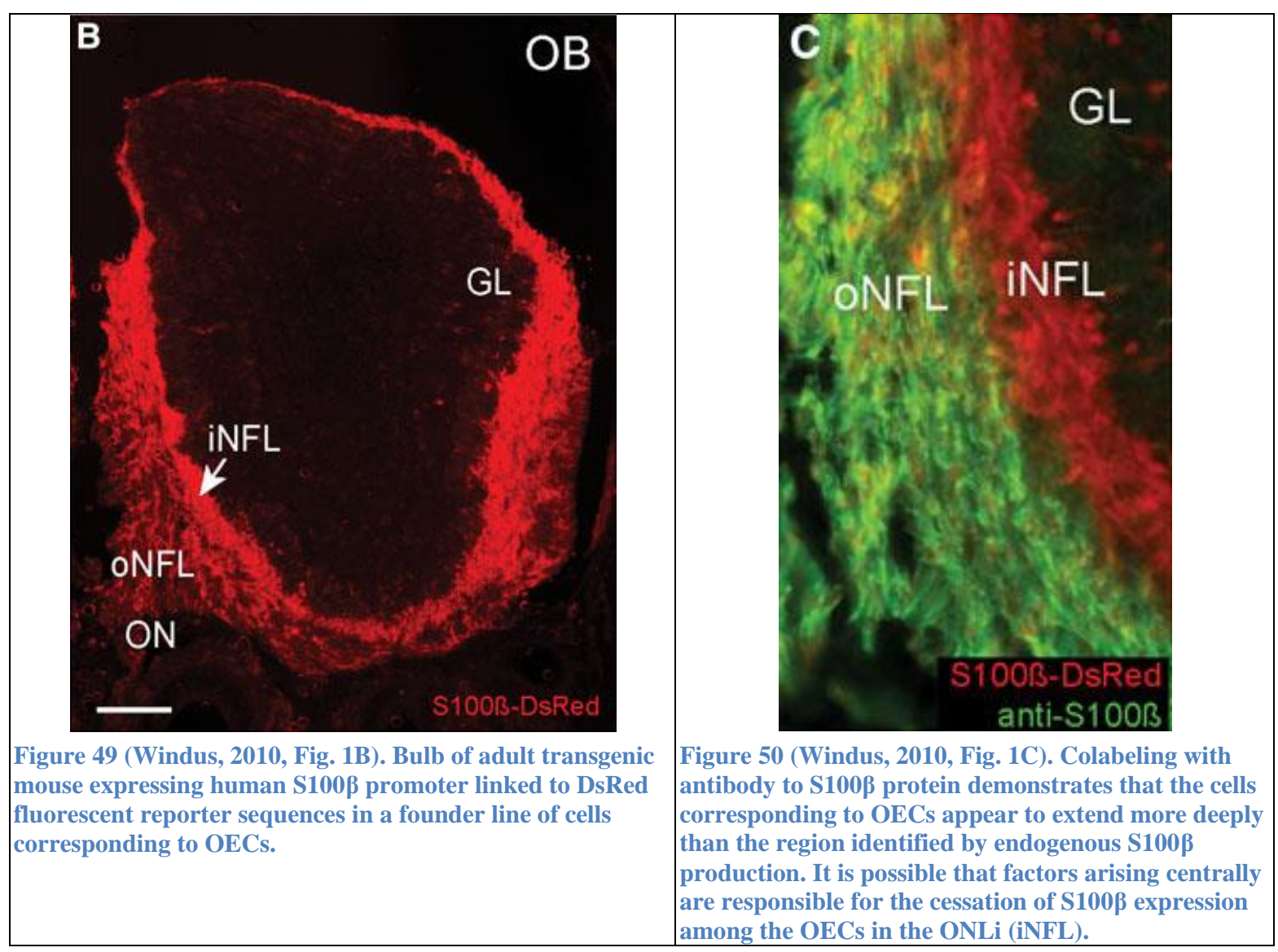

Even though the ectopic glomeruli experiments are limited in scope - only barely

resembling the full creation of the olfactory bulb - a possible implication is that the structure of the ONLo may not be functional per se, but arises as the result of factors unique to complete in vivo development.

We hypothesize that the ONLo forms during development as something more like an extension of the pattern of growth observed in the lamina propria. But here, rather than growing longitudinally from the epithelium to the telencephalon, the migratory units have grown over the spherical surface of the bulb. Then, the ONLi arises where the axons penetrate that surface, followed by p/OECs, and form the complex plexus as they reassort and coalesce into glomeruli. 


\section{Development of the bulb}

In mouse development, after E10 the axons and accompanying migratory cells leave the olfactory placode coincident with its invagination forming the olfactory pit (Cuschieri \& Bannister, 1975b; Doucette, 1989). Growing through the mesenchyme, the multiple groups of axons and migratory cells eventually coalesce into a "single, larger fascicle" near the rostral telencephalic vesicle that contains only axons and a homogeneous population of cells. This migratory mass first makes contact with the vesicle by E11.5 (Doucette, 1989; Hinds, 1972ab). It continues to grow dorso-caudally over the external glia limitans of the vesicle between E12 and E13. By E12, the axons have formed a thin distinctive layer on the surface of the cerebral vesicle, and by late E13 the vesicle has markedly evaginated at the regions of contact to form the primitive bulb. This "cap" of axons and p/OECs that is interposed between the primitive pial cells and the early glia limitans of the telencephalic vesicle is referred to as the presumptive ONL (Doucette, 1989). Valverde (1992) also makes the observation at this stage of the majority of axons staying on the surface of the bulb for a certain waiting period.

Gradually and beginning at the rostral end of the vesicle, individual and small groups of axons begin to penetrate the early basal lamina and enter the marginal zone of the bulb. They are initially unaccompanied by p/OECs. Eventually, the increasing number of these perforations create large disruptions in the basal lamina and allows p/OECs to resume following the axons (Figure 51, Figure 52; Marin-Padilla \& Amieva, 1989; Doucette, 1989). At about the same time the $\mathrm{p} / \mathrm{OECs}$ on the external border of the presumptive ONL produce a basal lamina and reestablish the glia limitans, and this results in the definitive ONL (Doucette, 1993a).

The presumptive ONL was defined on the basis of the mass of axons and p/OECs being separated from the telencephalic vesicle (or presumptive olfactory bulb) by an intact glia 
limitans. The definitive ONL was defined as the active elimination of the barrier between these and the deeper cellular constituents of the bulb primordium (Doucette, 1989, 1991, 1993a; Marin-Padilla \& Amieva, 1989), and when a new glia limitans was constituted by the p/OECs at the external border. As such, the presumptive and definitive ONL were the same cellular area, defined apart only by which surface faced a limiting membrane.

However, in these developmental studies, the ONL did not appear distinctly laminated ${ }^{5}$, and it was thought that the axons penetrating the early glia limitans were entering the presumptive glomerular layer. Since OECs appear to extensively migrate into this area (Figure 52), and these studies did not observe the complex morphology of an axonal plexus in the presumptive ONL, it is very probable that the formation of the ONLi begins inside of what was the early glia limitans.

Consequently, we postulate that the presumptive ONL defines the structure of the ONLo, and the definitive ONL should be redefined to encompass what forms outside the early glia limitans (the ONLo) and what forms inside of it (the ONLi).

There are two items of developmental evidence for equating the presumptive ONL with the eventual ONLo. First, the heterotypic nature of the bundles in the ONLo resembles those throughout the lamina propria. There should not be anything characteristically different in the mixed constituency of individual bundles during their development through the early mesenchyme - whether it be through the lamina propria or over the external surface of the early telencephalon. Second, the orientation of these bundles in the ONLo reflects the described formation of the presumptive ONL. While, in the lamina propria the bundles were extending more-or-less longitudinally towards the telencephalon, once they arrive, they do not uniformly and immediately penetrate its surface, but are described grow over it. Such parallel-to-surface 
spherical growth matches the fiber pattern in the ONLo. It is also possible that the ensuing evagination of the rostral telencephalic vesicle "ballooning" underneath this layer further draws the structure into such a shape.

Then, the real business of defasciculation, "the complex sampling of the environment", and "coalescence into glomeruli" takes place after the axons penetrate the early glia limitans. This area, then, would be not just the presumptive glomerular layer that Doucette (1989) describes - but it is first the ONLi, and this gives rise to the GL.

Many possibilities exist as to the specific mechanisms that would lead to the formation of the early ONLi/GL here and not more superficially. These may involve any combination of: (1) the initial lag of accompanying p/OECs past the early glia limitans, (2) spatial factors resulting here from the focal convergence of axons from the entire epithelial sheet, (3) induction by factors from within the telencephalon, or (4) molecular features intrinsic to specific subsets of axons or OECs that come into play at this proximity to the bulb. The last of these seems at least to be a requirement, as the ectopic glomeruli experiments required the transplantation of the entire olfactory sheet, and not just parts of it, in order to form glomeruli (Graziadei \& Monti-Graziadei, 1986).

In summary, here it has been proposed that the accumulation of axons on the surface of telencephalon during development - the presumptive ONL - defines the structure of the eventual ONLo, and the ONLi and GL form interiorly after the axons penetrate its surface and are followed by OECs. 


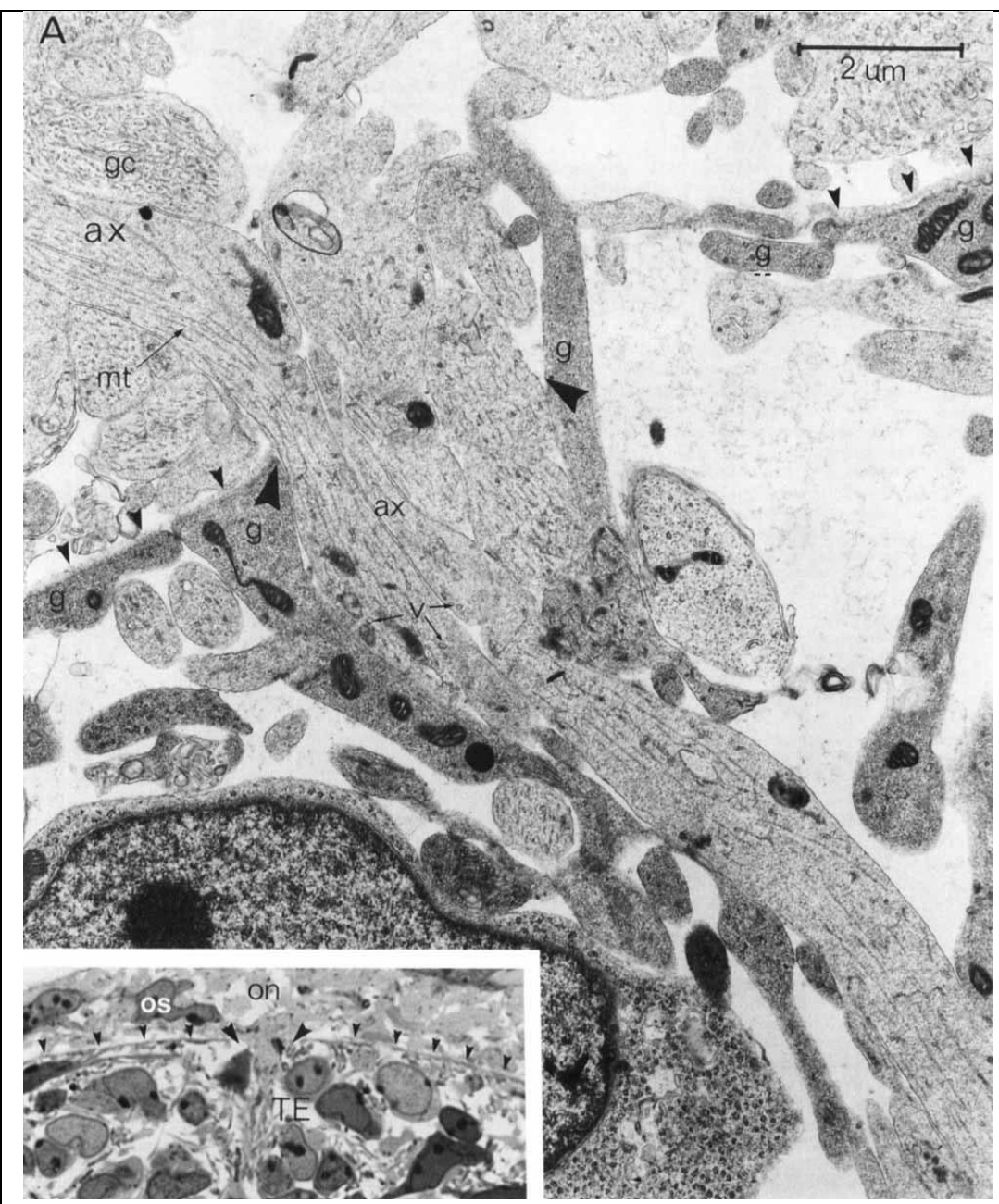

Figure 51 (Marin-Padilla \& Amieva, 1989, Fig. 6).

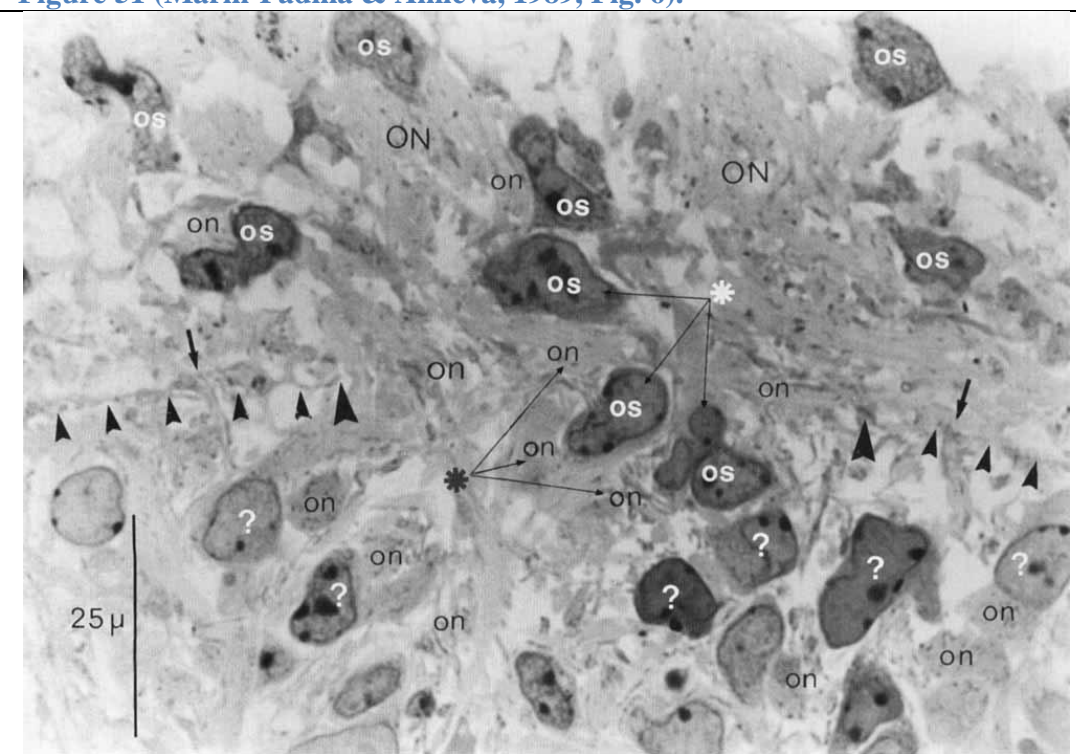

Figure 52 (Marin-Padilla \& Amieva, 1989, Fig. 7).
"Ultrastructural detail of a small perforation $(2.5 \mu \mathrm{m})$ of the telencephalic surface by a group of olfactory nerve axons showing the basal lamina opening (large arrowheads), the elevation of neighboring marginal glial processes (g), and the still intact surrounding surface (small arrowheads) of the CNS. The same area is depicted, at a light microscopic level, in the inset showing the direct contact (without intervening meningeal elements) of the parent olfactory nerve fascicle (on) with the CNS surface, the small basal lamina perforation (large arrowheads), the still intact large areas of the telencephalic surface (small arrowheads), and the presence of olfactory sheath cells (os) in the fascicle."
${ }^{6}$ Detail of a large disruption (ca. 60 $\mu \mathrm{m})$ in the surface of the embryonic telencephalon (large arrowheads) through which olfactory nerve axons (black *) with their accompanying olfactory sheath cells (white *) have entered into the nervous tissue. There is no anatomical discontinuity between these penetrating olfactory nerves (on) and sheath cells (os) with those of the above nerve fascicle that have established a direct contact with the CNS surface (small arrowheads). Below the surface of the telencephalon, and within the central nervous tissue, there are other recognizable olfactory nerve axons (on) with accompanying cells (?) which could also represent olfactory sheath cells. Two other small perforations of the telencephalic surface by individual olfactory nerve axons are also illustrated (small arrows). From ca. 12-day-old embryos." 


\section{6 - Glomerular Layer}

There is some controversy as to whether OECs are present in the GL enwrapping the homotypic bundles as they dive towards the entrance to individual glomeruli. Various studies using common biomarkers have asserted their absence (Au, 2002; Treloar, 1999), whereas electron-microscopic studies (Raisman, 1985; Valverde, 1991) have identified OECs on a structural basis, accompanying the axons until their entrance into glomeruli.

It would seem that the development of glomeruli by the coalescence of like-axons should not inherently preclude OECs from their continued accompaniment right up until the point of entrance to individual glomeruli. Rather, this may make it such that the OECs in the GL are not present en masse, forming a matrix as they do in the ONL, but rather tightly follow the specific coalesced bundles the short distance from the ONLi border into the individual glomeruli. So even if the OECs do continue to express a particular biomarker, such a limited presence may account for an inability to resolve OECs immunochemically at the light-microscopic level.

Electron microscopy has demonstrated ensheathing cells within the GL. Raisman (1985) observed such cells in the accessory olfactory bulb accompanying bundles of vomeronasal axons right up to the glomerular capsule. However, these were termed "superficial glia" (SG) because they had a more electron-dense appearance than the astrocytes forming the rest of the capsule. This allowed their being visually distinguishable from astrocytes, and enabled the detailed description of the glomerular capsule and synaptic zone. In the main olfactory bulb, Raisman notes, this contrast between the ensheathing cells and the astrocytes does not exist.

Another electron-microscopic examination of the main olfactory bulb in the hedgehog (Valverde, 1991) also demonstrated ensheathing cells following axons entering into the GL. The olfactory system in hedgehogs is highly developed, and there are multiple layers of glomeruli in 
the GL. This provided the opportunity to observe processes ensheathing the axons, passing between superficial glomeruli, and entering well into the GL to reach glomeruli situated more deeply.

Whether the ensheathing cells observed here are in fact OECs, rather than cells arising centrally, awaits further investigation. A definitive result could be provided with a immunoelectron-microscopic examination of this region in the aforementioned DsRed-S100 $\beta$ transgenic mouse.

In summary, the manner in which OECs accompany the olfactory axons throughout their development and throughout every compartment would seem to lend a high likelihood that they are present until their termination in synapses. Taken together with the formation of ectopic axonal plexuses and glomeruli-like structures being analogous to the development of the ONLi and olfactory glomeruli, there would seem to be no cause for OECs to cease ensheathing the axons until glomeruli actually form. It may be that after the formation of early glomeruli, and anchoring synapses with higher-order neurons (Malun \& Brunjes, 1996), a generalized "push" from astrocytes and other cells arising centrally causes the smooth contour apparent between the GL and the ONLi. But this, perhaps, does not strip away the OECs already ensheathing the bundles entering into glomeruli. Such a later-stage migration of astrocytes could also account for the interfascicular astrocytes observed in the ONL postnatally. 


\section{7 - Endnotes}

$1 \quad$ While the vomeronasal and olfactory nerves are often studied as analogous, they do differ in many ways. A review by Salazar (2009) considers the risk in extrapolating anatomical data on the vomeronasal system from species to species as it is a system which undergoes involution and regression with a great deal of variability among even closely related species. The main olfactory bulb is relatively consistent among mammalian species, thus any extrapolation from the accessory bulb to the main olfactory bulb of the same species bears a similar type of risk.

These complications may extend to aspects of the anatomy and cellular biology of the vomeronasal nerves as well. An olfactory-specific way to study the spatial dependence of cooperative ensheathment may be to compare the main olfactory nerves of various fishes - which range in length from millimeters in zebrafish, to 5.5-20 centimeters in European pike and garfish (Kreutzberg, 1977; Easton, 1971).

2 Figure 27 is approximately $3200 \mu \mathrm{m}^{2}$ and Figure 15 is approximately $20 \mu \mathrm{m}^{2}$. Thus, the former is 160 times larger than the latter.

$3 \quad$ None of the embryonic studies describe the presence of ONFs during the early development of the olfactory nerves. This in itself may tend to support their being last in the sequence. In addition, ONFs are not reported to be present in the migratory mass. When the relevant cells of the migratory mass - axons and pOECs - arrive at the surface of the embryonic telencephalon, they interpose themselves between the early glia limitans and the meninx primitiva. Were there mesodermal fibroblasts in this migratory mass, these would presumably develop into a cell type that differs from the LMCs of the pia mater, and be situated nearer the bulb than the LMCs. This is not observed, so it is not likely that fibroblasts are within the migratory mass. Thus, their relevance to the olfactory nerves arises as cells outside the original cast of cells in the olfactory system, and makes it likely that their role would come in last.

$4 \quad$ The data on the macaque is inconclusive. Nonetheless, we see that all species eventually exhibit the features of total merger in the ONL.

5 The failure to observe ONL laminarity in these developmental studies may also be due to the locations of the bulb examined, the time points examined, and/or the high-magnification of electron-microscopy reducing the likelihood of observing distinct laminae in a given view. 


\section{8 - References}

1. Astic L, Pellier-Monnin V, Godinot F. 1998. Spatio-temporal patterns of ensheathing cell differentiation in the rat olfactory system during development. Neuroscience 84:295-307.

2. Au WW, Treloar HB, Greer CA. 2002. Sublaminar organization of the mouse olfactory bulb nerve layer. J Comp Neurol 446:68-80.

3. Barber PC, Lindsay RM. 1982. Schwann cells of the olfactory nerves contain glial fibrillary acidic protein and resemble astrocytes. Neuroscience 7:3077-3090.

4. Bhatnagar KP, Kallen FC. 1974. Cribriform plate of ethmoid, olfactory bulb and olfactory acuity in forty species of bats. J Morphol 142:71-89.

5. Boyd JG, Skihar V, Kawaja M, Doucette R. 2003. Olfactory ensheathing cells: historical perspective and therapeutic potential. Anat Rec B New Anat 271:49-60.

6. Cao L, Su Z, Zhou Q, Lv B, Liu X, Jiao L, Li Z, Zhu Y, Huang Z, Huang A, He C. 2006. Glial cell line-derived neurotrophic factor promotes olfactory ensheathing cells migration. Glia 54:536-544.

7. Chehrehasa F, St John JA, Key B. 2006. Implantation of a scaffold following bulbectomy induces laminar organization of regenerating olfactory axons. Brain Res 1119:58-64.

8. Chuah MI, Au C. 1991. Olfactory Schwann cells are derived from precursor cells in the olfactory epithelium. J Neurosci Res 29:172-180.

9. Chuah MI, Au C. 1994. Olfactory cell cultures on ensheathing cell monolayers. Chem Senses 19:25-34.

10. Colombo JA, Napp MI, Puissant V. 1994. Leptomeningeal and skin fibroblasts: two different cell types? Int J Dev Neurosci 12:57-61.

11. Couly GF, Le Douarin NM. 1985. Mapping of the early neural primordium in quail-chick chimeras. I. Developmental relationships between placodes, facial ectoderm, and prosencephalon. Dev Biol 110:422-439.

12. Cuschieri A, Bannister LH. 1975. The development of the olfactory mucosa in the mouse: electron microscopy. J Anat 119:471-498.

13. Cuschieri A, Bannister LH. 1975. The development of the olfactory mucosa in the mouse: light microscopy. J Anat 119:277-286.

14. De Carlos JA, Lopez-Mascaraque L, Valverde F. 1995. The telencephalic vesicles are innervated by olfactory placode-derived cells: a possible mechanism to induce neocortical development. Neuroscience 68:1167-1178.

15. Deumens R, Koopmans GC, Den Bakker CG, Maquet V, Blacher S, Honig WM, Jerome R, Pirard JP, Steinbusch HW, Joosten EA. 2004. Alignment of glial cells stimulates directional neurite growth of CNS neurons in vitro. Neuroscience 125:591-604.

16. Deumens R, Koopmans GC, Honig WM, Hamers FP, Maquet V, Jerome R, Steinbusch HW, Joosten EA. 2006. Olfactory ensheathing cells, olfactory nerve fibroblasts and biomatrices to promote long-distance axon regrowth and functional recovery in the dorsally hemisected adult rat spinal cord. Exp Neurol 200:89-103. 
17. Doucette JR. 1984. The glial cells in the nerve fiber layer of the rat olfactory bulb. Anat Rec 210:385-391.

18. Doucette JR, Kiernan JA, Flumerfelt BA. 1983. The re-innervation of olfactory glomeruli following transection of primary olfactory axons in the central or peripheral nervous system. J Anat 137 (Pt 1):1-19.

19. Doucette JR, Kiernan JA, Flumerfelt BA. 1983. Two different patterns of retrograde degeneration in the olfactory epithelium following transection of primary olfactory axons. $\mathrm{J}$ Anat 136:673-689.

20. Doucette R. 1989. Development of the nerve fiber layer in the olfactory bulb of mouse embryos. J Comp Neurol 285:514-527.

21. Doucette R. 1990. Glial influences on axonal growth in the primary olfactory system. Glia 3:433-449.

22. Doucette R. 1991. PNS-CNS transitional zone of the first cranial nerve. J Comp Neurol 312:451-466.

23. Doucette R. 1993. Glial cells in the nerve fiber layer of the main olfactory bulb of embryonic and adult mammals. Microsc Res Tech 24:113-130.

24. Doucette R. 1993. Glial progenitor cells of the nerve fiber layer of the olfactory bulb: effect of astrocyte growth media. J Neurosci Res 35:274-287.

25. Easton DM. 1971. Garfish olfactory nerve: easily accessible source of numerous long, homogeneous, nonmyelinated axons. Science 172:952-955.

26. Farbman AI. 1992. Cell biology of olfaction. Cambridge ; New York, N.Y., USA: Cambridge University Press.

27. Farbman AI, Squinto LM. 1985. Early development of olfactory receptor cell axons. Brain Res 351:205-213.

28. Feinstein P, Mombaerts P. 2004. A contextual model for axonal sorting into glomeruli in the mouse olfactory system. Cell 117:817-831.

29. Field P, Li Y, Raisman G. 2003. Ensheathment of the olfactory nerves in the adult rat. J Neurocytol 32:317-324.

30. Fraher JP. 1982. The ultrastructure of sheath cells in developing rat vomeronasal nerve. J Anat 134:149-168.

31. Franceschini IA, Barnett SC. 1996. Low-affinity NGF-receptor and E-N-CAM expression define two types of olfactory nerve ensheathing cells that share a common lineage. Dev Biol 173:327-343.

32. Franssen EH, de Bree FM, Verhaagen J. 2007. Olfactory ensheathing glia: their contribution to primary olfactory nervous system regeneration and their regenerative potential following transplantation into the injured spinal cord. Brain Res Rev 56:236-258.

33. Gasser HS. 1956. Olfactory nerve fibers. J Gen Physiol 39:473-496. 
34. Gong Q, Bailey MS, Pixley SK, Ennis M, Liu W, Shipley MT. 1994. Localization and regulation of low affinity nerve growth factor receptor expression in the rat olfactory system during development and regeneration. J Comp Neurol 344:336-348.

35. Goodman MN, Silver J, Jacobberger JW. 1993. Establishment and neurite outgrowth properties of neonatal and adult rat olfactory bulb glial cell lines. Brain Res 619:199-213.

36. Graziadei PP, DeHan RS. 1973. Neuronal regeneration in frog olfactory system. J Cell Biol 59:525-530.

37. Graziadei PP, Kaplan MS. 1980. Regrowth of olfactory sensory axons into transplanted neural tissue. 1. Development of connections with the occipital cortex. Brain Res 201:39-44.

38. Graziadei PP, Karlan MS, Graziadei GA, Bernstein JJ. 1980. Neurogenesis of sensory neurons in the primate olfactory system after section of the fila olfactoria. Brain Res 186:289-300.

39. Graziadei PP, Levine RR, Graziadei GA. 1978. Regeneration of olfactory axons and synapse formation in the forebrain after bulbectomy in neonatal mice. Proc Natl Acad Sci U S A 75:5230-5234.

40. Graziadei PP, Levine RR, Monti Graziadei GA. 1979. Plasticity of connections of the olfactory sensory neuron: regeneration into the forebrain following bulbectomy in the neonatal mouse. Neuroscience 4:713-727.

41. Graziadei PP, Monti Graziadei GA. 1980. Neurogenesis and neuron regeneration in the olfactory system of mammals. III. Deafferentation and reinnervation of the olfactory bulb following section of the fila olfactoria in rat. J Neurocytol 9:145-162.

42. Graziadei PP, Monti Graziadei GA. 1986. Principles of organization of the vertebrate olfactory glomerulus: an hypothesis. Neuroscience 19:1025-1035.

43. Graziadei PP, Samanen DW. 1980. Ectopic glomerular structures in the olfactory bulb of neonatal and adult mice. Brain Res 187:467-472.

44. Hamlin JA, Fang H, Schwob JE. 2004. Differential expression of the mammalian homologue of fasciclin II during olfactory development in vivo and in vitro. J Comp Neurol 474:438452.

45. Herrera LP, Casas CE, Bates ML, Guest JD. 2005. Ultrastructural study of the primary olfactory pathway in Macaca fascicularis. J Comp Neurol 488:427-441.

46. Hinds JW. 1972. Early neuron differentiation in the mouse of olfactory bulb. I. Light microscopy. J Comp Neurol 146:233-252.

47. Hinds JW. 1972. Early neuron differentiation in the mouse olfactory bulb. II. Electron microscopy. J Comp Neurol 146:253-276.

48. Hinds JW, McNelly NA. 1981. Aging in the rat olfactory system: correlation of changes in the olfactory epithelium and olfactory bulb. J Comp Neurol 203:441-453.

49. Holbrook EH, Leopold DA, Schwob JE. 2005. Abnormalities of axon growth in human olfactory mucosa. Laryngoscope 115:2144-2154. 
50. Holbrook EH, Szumowski KE, Schwob JE. 1995. An immunochemical, ultrastructural, and developmental characterization of the horizontal basal cells of rat olfactory epithelium. $\mathbf{J}$ Comp Neurol 363:129-146.

51. Johnson EW. 2000. Immunocytochemical characteristics of cells and fibers in the nasal mucosa of young and adult macaques. Anat Rec 259:215-228.

52. Julliard AK, Hartmann DJ. 1998. Spatiotemporal patterns of expression of extracellular matrix molecules in the developing and adult rat olfactory system. Neuroscience 84:11351150 .

53. Kawaja MD, Boyd JG, Smithson LJ, Jahed A, Doucette R. 2009. Technical strategies to isolate olfactory ensheathing cells for intraspinal implantation. J Neurotrauma 26:155-177.

54. Kreutzberg GW, Gross GW. 1977. General morphology and axonal ultrastructure of the olfactory nerve of the pike, Esox lucius. Cell Tissue Res 181:443-457.

55. Li Y, Decherchi P, Raisman G. 2003. Transplantation of olfactory ensheathing cells into spinal cord lesions restores breathing and climbing. J Neurosci 23:727-731.

56. Li Y, Field PM, Raisman G. 1997. Repair of adult rat corticospinal tract by transplants of olfactory ensheathing cells. Science 277:2000-2002.

57. Li Y, Field PM, Raisman G. 1998. Regeneration of adult rat corticospinal axons induced by transplanted olfactory ensheathing cells. J Neurosci 18:10514-10524.

58. Li Y, Field PM, Raisman G. 2005. Olfactory ensheathing cells and olfactory nerve fibroblasts maintain continuous open channels for regrowth of olfactory nerve fibres. Glia $52: 245-251$.

59. Li Y, Li D, Raisman G. 2005. Interaction of olfactory ensheathing cells with astrocytes may be the key to repair of tract injuries in the spinal cord: the 'pathway hypothesis'. J Neurocytol 34:343-351.

60. Liu KL, Chuah MI, Lee KK. 1995. Soluble factors from the olfactory bulb attract olfactory Schwann cells. J Neurosci 15:990-1000.

61. Malun D, Brunjes PC. 1996. Development of olfactory glomeruli: temporal and spatial interactions between olfactory receptor axons and mitral cells in opossums and rats. J Comp Neurol 368:1-16.

62. Marin-Padilla M, Amieva MR. 1989. Early neurogenesis of the mouse olfactory nerve: Golgi and electron microscopic studies. J Comp Neurol 288:339-352.

63. Menco BP. 1980. Qualitative and quantitative freeze-fracture studies on olfactory and nasal respiratory structures of frog, ox, rat, and dog. I. A general survey. Cell Tissue Res 207:183209.

64. Mombaerts P, Wang F, Dulac C, Chao SK, Nemes A, Mendelsohn M, Edmondson J, Axel R. 1996. Visualizing an olfactory sensory map. Cell 87:675-686.

65. Norgren RB, Jr., Ratner N, Brackenbury R. 1992. Development of olfactory nerve glia defined by a monoclonal antibody specific for Schwann cells. Dev Dyn 194:231-238.

66. O'Rahilly R, Muller F. 1986. The meninges in human development. J Neuropathol Exp Neurol 45:588-608. 
67. Pihlstrom H, Fortelius M, Hemila S, Forsman R, Reuter T. 2005. Scaling of mammalian ethmoid bones can predict olfactory organ size and performance. Proc Biol Sci 272:957-962.

68. Potter SM, Zheng C, Koos DS, Feinstein P, Fraser SE, Mombaerts P. 2001. Structure and emergence of specific olfactory glomeruli in the mouse. J Neurosci 21:9713-9723.

69. Rafols JA, Getchell TV. 1983. Morphological relations between the receptor neurons, sustentacular cells and Schwann cells in the olfactory mucosa of the salamander. Anat Rec 206:87-101.

70. Raisman G. 1985. Specialized neuroglial arrangement may explain the capacity of vomeronasal axons to reinnervate central neurons. Neuroscience 14:237-254.

71. Raisman G. 2001. Olfactory ensheathing cells - another miracle cure for spinal cord injury? Nat Rev Neurosci 2:369-375.

72. Ramon-Cueto A, Avila J. 1998. Olfactory ensheathing glia: properties and function. Brain Res Bull 46:175-187.

73. Rela L, Bordey A, Greer CA. 2010. Olfactory ensheathing cell membrane properties are shaped by connectivity. Glia 58:665-678.

74. Salazar I, Quinteiro PS. 2009. The risk of extrapolation in neuroanatomy: the case of the Mammalian vomeronasal system. Front Neuroanat 3:22.

75. Schwob JE, Szumowski KE, Stasky AA. 1992. Olfactory sensory neurons are trophically dependent on the olfactory bulb for their prolonged survival. J Neurosci 12:3896-3919.

76. Skinner AP, Pachnicke S, Lakatos A, Franklin RJ, Jeffery ND. 2005. Nasal and frontal sinus mucosa of the adult dog contain numerous olfactory sensory neurons and ensheathing glia. Res Vet Sci 78:9-15.

77. Smithson LJ, Kawaja MD. 2009. A comparative examination of biomarkers for olfactory ensheathing cells in cats and guinea pigs. Brain Res 1284:41-53.

78. St John JA, Clarris HJ, McKeown S, Royal S, Key B. 2003. Sorting and convergence of primary olfactory axons are independent of the olfactory bulb. J Comp Neurol 464:131-140.

79. St John JA, Key B. 2001. Chemically and morphologically identifiable glomeruli in the rat olfactory bulb. J Comp Neurol 436:497-507.

80. Storan MJ, Key B. 2004. Target tissue influences the peripheral trajectory of mouse primary sensory olfactory axons. J Neurobiol 61:175-188.

81. Suzuki Y, Takeda M, Farbman AI. 1996. Supporting cells as phagocytes in the olfactory epithelium after bulbectomy. J Comp Neurol 376:509-517.

82. Tennent R, Chuah MI. 1996. Ultrastructural study of ensheathing cells in early development of olfactory axons. Brain Res Dev Brain Res 95:135-139.

83. Tisay KT, Key B. 1999. The extracellular matrix modulates olfactory neurite outgrowth on ensheathing cells. J Neurosci 19:9890-9899.

84. Treloar HB, Feinstein P, Mombaerts P, Greer CA. 2002. Specificity of glomerular targeting by olfactory sensory axons. J Neurosci 22:2469-2477. 
85. Treloar HB, Nurcombe V, Key B. 1996. Expression of extracellular matrix molecules in the embryonic rat olfactory pathway. J Neurobiol 31:41-55.

86. Treloar HB, Purcell AL, Greer CA. 1999. Glomerular formation in the developing rat olfactory bulb. J Comp Neurol 413:289-304.

87. Valverde F, Lopez-Mascaraque L. 1991. Neuroglial arrangements in the olfactory glomeruli of the hedgehog. J Comp Neurol 307:658-674.

88. Valverde F, Santacana M, Heredia M. 1992. Formation of an olfactory glomerulus: morphological aspects of development and organization. Neuroscience 49:255-275.

89. Wewetzer K, Kern N, Ebel C, Radtke C, Brandes G. 2005. Phagocytosis of O4+ axonal fragments in vitro by p75- neonatal rat olfactory ensheathing cells. Glia 49:577-587.

90. Whitlock KE. 2004. A new model for olfactory placode development. Brain Behav Evol 64:126-140.

91. Windus LC, Claxton C, Allen CL, Key B, St John JA. 2007. Motile membrane protrusions regulate cell-cell adhesion and migration of olfactory ensheathing glia. Glia 55:1708-1719.

92. Windus LC, Lineburg KE, Scott SE, Claxton C, Mackay-Sim A, Key B, St John JA. 2010. Lamellipodia mediate the heterogeneity of central olfactory ensheathing cell interactions. Cell Mol Life Sci 67:1735-1750. 